\title{
Asymmetric variations of multifunctions with application to functional inclusions
}

\author{
Vyacheslav V. Chistyakov*,a \\ ${ }^{a}$ Department of Informatics, Mathematics and Computer Science, \\ National Research University Higher School of Economics, \\ Bol'shaya Pechërskaya Street 25/12, Nizhny Novgorod \\ 603155, Russia
}

\begin{abstract}
Under certain initial conditions, we prove the existence of set-valued selectors of univariate compact-valued multifunctions of bounded (Jordan) variation when the notion of variation is defined taking into account only the Pompeiu asymmetric excess between compact sets from the target metric space. For this, we study subtle properties of the directional variations. We show by examples that all assumptions in the main existence result are essential. As an application, we establish the existence of set-valued solutions $X(t)$ of bounded variation to the functional inclusion of the form $X(t) \subset F(t, X(t))$ satisfying the initial condition $X\left(t_{0}\right)=X_{0}$.
\end{abstract}

Key words: multifunction, bounded variation, compact set, Hausdorff metric, Pompeiu excess, set-valued selector, selection principle MSC 2000: 54C65, 54C60, 26A45, 54D30

\section{Introduction}

This paper is devoted to the existence of (set-valued) selectors (or selections) with prescribed initial conditions of multifunctions (=set-valued mappings) with compact images from a metric space. Continuous and Lipschitz continuous selectors exist, in general, for multifunctions with convex (and closed) images from a Banach space $([2,28,31])$. Many examples are known

\footnotetext{
${ }^{*}$ Corresponding author.

Email address: czeslaw@mail.ru, vchistyakov@hse.ru (Vyacheslav V. Chistyakov)
} 
when a continuous (or even Hölder continuous) multifunction from a closed interval $T=[a, b] \subset \mathbb{R}$ into a family of compact subsets of a ball in $\mathbb{R}^{2}$, or a Lipschitz continuous multifunction from $\mathbb{R}^{3}$ into a family of compact subsets of a ball in $\mathbb{R}^{3}$ have no continuous selector $([1,15,[23,[26,28])$.

In contrast to this, it was shown in 24] that a Lipschitz continuous multifunction $F$ on $[a, b]$ with compact images from $\mathbb{R}^{N}$ admits a Lipschitz continuous selector, whose Lipschitz constant does not exceed that of the multifunction; furthermore, if $F$ is only continuous and of bounded (Jordan) variation, then it admits a continuous selector. Similar assertions for Lipschitz and absolutely continuous multifunctions with convex and nonconvex images from $\mathbb{R}^{N}$ were established in [22, 25, 27, 35]. The selector results from [24] were extended in [29, Suppl. 1] for multifunctions $F$ on $[a, b]$ with compact graphs and images in a Banach space, and in [33] for metric space valued multifunctions with compact images. It is to be noted that the compactness arguments in these references were based on Arzelà-Ascoli's theorem.

Changing the compactness arguments to generalized Helly's pointwise selection principle, it was proved in [8] that a multifunction $F$ on $[a, b]$ of bounded Jordan variation with compact images from a Banach space admits a selector, whose total Jordan variation does not exceed that of $F$ and passes through a given point in the graph of $F$. Thus, "nice" selectors of compact-valued multifunctions inherit boundedness of variation rather than continuity $([13])$. The results of [8] were then extended to multifunctions with compact images in a metric space and having certain regularity such as absolute continuity, Lipschitz continuity, boundedness of Riesz-OrliczMedvedev generalized variation, boundedness of essential variation, measurability in the first variable and boundedness of variation in the second variable $([3,99,10,11,12,13,16,18])$. The existence of selections of bounded variation was applied to the study of set-valued measure differential problems ([32]).

The notion of bounded variation of a multifunction $F$ from a subset $T \subset \mathbb{R}$ into a family of compact subsets of a metric space relies on the linear order in the domain $T$ of $F$ and the Pompeiu-Hausdorff metric in the range of $F$, which is the maximum of two asymmetric excesses (cf. Section 2). In this paper, we prove the existence of set-valued selectors (in particular, singlevalued selectors) of bounded Jordan variation (with respect to the PompeiuHausdorff metric) under milder assumptions of boundedness of directional variations of $F$, to the right or to the left, with respect to the Pompeiu excess only ([17] and Section 2). Our main result, Theorem 1 in Section 2, extends the results of [17] for single-valued selectors, and some partial results 
of [34] concerning set-valued selectors.

This paper, along with [17], may be considered as part of analysis in asymmetric spaces, giving an intuition of the notion of bounded variation in the case when the distance function on the underlying space does not satisfy the symmetry axiom (for asymmetric analysis in normed spaces, cf. [19]).

The paper is organized as follows. In Section 2, we review certain definitions and facts and present our main result, Theorem 1. Section 3 is devoted to the (subtle) properties of directional variations, presented for compact-valued multifunctions defined on an arbitrary subset of $\mathbb{R}$ (hence suitable for linearly ordered sets as the domains). In Section 4, we prove Theorem 1 and exhibit some of its consequences. We illustrate our result by suitable examples in Section 5. In Section 6, we prove the existence of set-valued solutions of bounded variation to the functional inclusion of the form $X(t) \subset F(t, X(t))$ for all $t \in T$.

\section{Main Result}

We begin by reviewing certain definitions and facts needed for our results. Throughout the paper $(M, d)$ is a metric space with metric $d$.

Given two nonempty sets $X, Y \subset M$, the Pompeiu excess (écart, in French) of $X$ over $Y$ is defined by ([30, pp. 281-282], [5, Chapter II])

$$
e(X, Y) \equiv e_{d}(X, Y):=\sup _{x \in X} d(x, Y)=\inf \left\{r>0: X \subset \mathcal{O}_{r}(Y)\right\}
$$

where $d(x, Y):=\inf _{y \in Y} d(x, y)$ is the distance 1 from $x \in M$ to $Y$, and the set $\mathcal{O}_{r}(Y):=\{x \in M: d(x, Y)<r\}$ is the open $r$-neighbourhood 2 of $Y, r>0$ (if $Y=\{y\}, \mathcal{O}_{r}(y) \equiv \mathcal{O}_{r}(Y)$ is simply the open ball of radius $r$ centered at $y \in M)$. Note that $e(X, Y) \neq e(Y, X)$ in general.

The well-known properties of $e(\cdot, \cdot)$ are as follows. Given $X, Y, Z \subset M$ :

(a) $e(X, Y)=0$ iff $X \subset \bar{Y}$, where $\bar{Y}=\bigcap_{r>0} \mathcal{O}_{r}(Y)$ is the closure of $Y$ in $M$;

(b) $e(X, Y) \leq e(X, Z)+e(Z, Y)$ (triangle inequality for $e$ );

(c) $e(X, Y)<+\infty$ provided $X$ and $Y$ are bounded (and, in particular bounded and closed, or compact).

\footnotetext{
${ }^{1} d(x, \varnothing):=+\infty$.

${ }^{2} \mathcal{O}_{r}(\varnothing):=\varnothing$ for $r>0 ; e(\varnothing, Y):=0$ for any $Y \subset M$, and $e(X, \varnothing):=+\infty$ if $\varnothing \neq X \subset M$.

'iff' means as usual 'if and only if'.
} 
The Pompeiu-Hausdorff distance between two sets $X, Y \subset M$ is defined by (e.g., [30], [5, Chapter II]) ${ }^{4}$

$d_{H}(X, Y):=\max \{e(X, Y), e(Y, X)\}=\inf \left\{r>0: X \subset \mathcal{O}_{r}(Y)\right.$ and $\left.Y \subset \mathcal{O}_{r}(X)\right\}$

It follows from (a), (b), and (c) above that $d_{H}$ is a metric (with finite values), called the Pompeiu-Hausdorff metric, on the family $\operatorname{bcl}(M)$ of all nonempty bounded closed subsets of $M$ and, in particular on the family $\mathrm{c}(M)$ of all nonempty compact subsets of $M$.

A multifunction (or set-valued mapping) from a nonempty set $T$ into $M$ is a rule $F$, which assigns to each $t \in T$ a unique subset $F(t) \subset M$; in symbols, $F: T \rightarrow \mathcal{P}(M)$, where $\mathcal{P}(M)$ is the power set of $M$ (= the family of all subsets of $M)$. A multifunction $\Gamma: T \rightarrow \mathcal{P}(M)$ is said to be a setvalued selector of $F: T \rightarrow \mathcal{P}(M)$ on $T$ provided $\Gamma(t) \subset F(t)$ for all $t \in T$. Clearly, $F$ is a set-valued selector of itself. If $\Gamma: T \rightarrow M$ is single-valued and $\Gamma(t) \in F(t)$ for all $t \in T$, then $\Gamma$ is called a selector (or selection) of $F$ on $T$.

Of main interest in this paper are multifunctions $F: T \rightarrow \mathrm{c}(M)$ with $T$ a nonempty subset of the reals $\mathbb{R}$. Such an $F$ is said to be of bounded variation (with respect to $d_{H}$ ) provided its (total) Jordan variation

$$
\mathrm{V}(F, T):=\sup _{\pi} \sum_{i=1}^{m} d_{H}\left(F\left(t_{i-1}\right), F\left(t_{i}\right)\right) \text { is finite } \quad(\mathrm{V}(F, \varnothing):=0),
$$

the supremum being taken over all partitions $\pi$ of the set $T \subset \mathbb{R}$, i.e., $m \in \mathbb{N}$ and $\pi=\left\{t_{i}\right\}_{i=0}^{m} \subset T$ such that $t_{i-1} \leq t_{i}$ for all $i \in\{1, \ldots, m\}$.

The following theorem on the existence of set-valued selectors of bounded variation was established in [13, Theorems 10.1 and 5.1]:

Theorem A. Given $T \subset \mathbb{R}, t_{0} \in T, X_{0} \in \mathrm{c}(M)$, and $F: T \rightarrow \mathrm{c}(M)$ such that $\mathrm{V}(F, T)<+\infty$, there exists a set-valued selector $\Gamma: T \rightarrow \mathrm{c}(M)$ of $F$ on $T$ such that $d_{H}\left(X_{0}, \Gamma\left(t_{0}\right)\right) \leq e\left(X_{0}, F\left(t_{0}\right)\right)$ and $\mathrm{V}(\Gamma, T) \leq \mathrm{V}(F, T)$. (In particular, if $X_{0} \subset F\left(t_{0}\right)$, the first inequality above gives $\Gamma\left(t_{0}\right)=X_{0}$.) Furthermore, if $x_{0} \in M$ and $X_{0}=\left\{x_{0}\right\}$, then $\Gamma: T \rightarrow M$ may be chosen to be single-valued and such that $d\left(x_{0}, \Gamma\left(t_{0}\right)\right) \leq d\left(x_{0}, F\left(t_{0}\right)\right)$.

Theorem A already contains as particular cases many previously known results [3], [8]- 12], [18, 22, 24, 29, 33], concerning single-valued selectors.

\footnotetext{
${ }^{4}$ Pompeiu [30] symmetrized the excess $e$ by $d_{P}(X, Y):=e(X, Y)+e(Y, X)$ (cf. also [4]).
} 
The purpose of this paper is to drop the assumption $\mathrm{V}(F, T)<+\infty$, replacing it by 'directional' ones $\overrightarrow{\mathrm{V}}(F, T)<+\infty$ or $\overleftarrow{\mathrm{V}}(F, T)<+\infty$ (see below), which, as will be shown, still guarantees the existence of set-valued selectors $\Gamma$ of $F$ on $T$ of bounded variation (with respect to $d_{H}$ ).

In order to do it, given $F: T \rightarrow \mathrm{c}(M)$, following [17] the quantities

$$
\overrightarrow{\mathrm{V}}(F, T):=\sup _{\pi} \sum_{i=1}^{m} e\left(F\left(t_{i-1}\right), F\left(t_{i}\right)\right) \text { and } \overleftarrow{\mathrm{V}}(F, T):=\sup _{\pi} \sum_{i=1}^{m} e\left(F\left(t_{i}\right), F\left(t_{i-1}\right)\right)
$$

are said to be the directional (or excess) variations of $F$ to the right and to the left, respectively $(\overrightarrow{\mathrm{V}}(F, \varnothing)=\overleftarrow{\mathrm{V}}(F, \varnothing):=0)$. Clearly,

$$
\max \{\overrightarrow{\mathrm{V}}(F, T), \overleftarrow{\mathrm{V}}(F, T)\} \leq \mathrm{V}(F, T) \leq \overrightarrow{\mathrm{V}}(F, T)+\overleftarrow{\mathrm{V}}(F, T),
$$

and so, $\mathrm{V}(F, T)$ is finite iff both $\overrightarrow{\mathrm{V}}(F, T)$ and $\overleftarrow{\mathrm{V}}(F, T)$ are finite. Note that if $F: T \rightarrow M$ is single-valued, the quantity $\mathrm{V}(F, T)=\overrightarrow{\mathrm{V}}(F, T)=\overleftarrow{\mathrm{V}}(F, T)$ is the usual (Jordan) variation of $F$ on $T$ (e.g., [9]).

Our first main result, extending Theorem $\mathrm{A}$, is the following theorem on the existence of set-valued selectors of bounded variation. For brevity, we write $T_{S}:=T \cap S$ for $S \subset \mathbb{R}$ (e.g., $T_{\left[t_{0},+\infty\right)}=T \cap\left[t_{0},+\infty\right)$ for $t_{0} \in T$, etc.).

Theorem 1. Suppose $T \subset \mathbb{R}, t_{0} \in T, X_{0} \in \mathrm{c}(M)$, and $F: T \rightarrow \mathrm{c}(M)$.

(a) Let $a:=\inf T \in T$. If $\overrightarrow{\mathrm{V}}(F, T)<+\infty$, then there exists a set-valued selector of bounded variation $\Gamma: T \rightarrow \mathrm{c}(M)$ of $F$ on $T$ such that $d_{H}\left(X_{0}, \Gamma\left(t_{0}\right)\right) \leq e\left(X_{0}, F\left(t_{0}\right)\right), \mathrm{V}\left(\Gamma, T_{\left[a, t_{0}\right)}\right) \leq \overrightarrow{\mathrm{V}}\left(F, T_{\left[a, t_{0}\right)}\right), \mathrm{V}\left(\Gamma, T_{\left[t_{0},+\infty\right)}\right)$ $\leq \overrightarrow{\mathrm{V}}\left(F, T_{\left[t_{0},+\infty\right)}\right)$, and

$$
\mathrm{V}(\Gamma, T)-J_{a}\left(\Gamma, t_{0}\right) \leq \overrightarrow{\mathrm{V}}\left(F, T_{\left[a, t_{0}\right)}\right)+\overrightarrow{\mathrm{V}}\left(F, T_{\left[t_{0},+\infty\right)}\right) \leq \overrightarrow{\mathrm{V}}(F, T),
$$

where $J_{a}\left(\Gamma, t_{0}\right):=0$ if $t_{0}=a$, and if $t_{0}>a$ and $s_{0}:=\sup T_{\left[a, t_{0}\right)}$,

$$
J_{a}\left(\Gamma, t_{0}\right):=\left\{\begin{array}{cll}
d_{H}\left(\Gamma\left(s_{0}\right), \Gamma\left(t_{0}\right)\right), & \text { if } & \left.s_{0} \in T_{\left[a, t_{0}\right.}\right) \\
\lim _{T \ni t \rightarrow s_{0}-0} \mathrm{~V}\left(\Gamma, T_{\left[t, t_{0}\right]}\right), & \text { if } & s_{0} \notin T_{\left[a, t_{0}\right)} .
\end{array}\right.
$$

In particular, if $s_{0}=t_{0}$, then $J_{a}\left(\Gamma, t_{0}\right)=\lim _{T \ni t \rightarrow t_{0}-0} d_{H}\left(\Gamma(t), \Gamma\left(t_{0}\right)\right)$.

(b) Let $b:=\sup T \in T$. If $\overleftarrow{\mathrm{V}}(F, T)<+\infty$, then there exists a set-valued selector of bounded variation $\Gamma: T \rightarrow \mathrm{c}(M)$ of $F$ on $T$ such that 


$$
\begin{aligned}
& d_{H}\left(X_{0}, \Gamma\left(t_{0}\right)\right) \leq e\left(X_{0}, F\left(t_{0}\right)\right), \mathrm{V}\left(\Gamma, T_{\left(t_{0}, b\right]}\right) \leq \overleftarrow{\mathrm{V}}\left(F, T_{\left(t_{0}, b\right]}\right), \mathrm{V}\left(\Gamma, T_{\left(-\infty, t_{0}\right]}\right) \\
& \leq \overleftarrow{\mathrm{V}}\left(F, T_{\left(-\infty, t_{0}\right]}\right) \text {, and } \\
& \qquad \begin{aligned}
\mathrm{V}(\Gamma, T)-J_{b}\left(\Gamma, t_{0}\right) \leq \overleftarrow{\mathrm{V}}\left(F, T_{\left(t_{0}, b\right]}\right)+\overleftarrow{\mathrm{V}}\left(F, T_{\left(-\infty, t_{0}\right]}\right) \leq \overleftarrow{\mathrm{V}}(F, T), \\
\text { where } J_{b}\left(\Gamma, t_{0}\right):=0 \text { if } t_{0}=b, \text { and if } t_{0}<b \text { and } s_{0}:=\inf T_{\left(t_{0}, b\right]},
\end{aligned} \\
& \qquad J_{b}\left(\Gamma, t_{0}\right):=\left\{\begin{array}{c}
d_{H}\left(\Gamma\left(t_{0}\right), \Gamma\left(s_{0}\right)\right), \\
\lim _{T \ni t \rightarrow s_{0}+0} \mathrm{~V}\left(\Gamma, T_{\left[t_{0}, t\right]}\right), \quad \text { if } \quad s_{0} \in T_{\left(t_{0}, b\right]} \notin T_{\left(t_{0}, b\right]} .
\end{array}\right.
\end{aligned}
$$

In particular, if $s_{0}=t_{0}$, then $J_{b}\left(\Gamma, t_{0}\right)=\lim _{T \ni t \rightarrow t_{0}+0} d_{H}\left(\Gamma\left(t_{0}\right), \Gamma(t)\right)$.

This theorem will be proved in Section 4, where it will also be shown that Theorem 1 implies Theorem A. A somewhat free and intuitive interpretation of Theorem 1 can be given as follows. We may imagine $F$ to be a road with the value $F(t)$ as a section at a given coordinate $t$. If $t$ increases, the section $F(t)$ moves in one direction, say, to the right. Theorem 1 asserts that if the road $F$ is properly built/controlled (i.e., its variation to the right is finite), then an extended object (e.g., a car) can freely pass it.

\section{Properties of Directional Variations}

In this section, we gather auxiliary facts needed for the proof of Theorem 1. Let $T \subset \mathbb{R}$ and $F: T \rightarrow \mathrm{c}(M)$ be a multifunction.

\subsection{Monotonicity of $F$}

By the definition of $\mathrm{V}, \mathrm{V}(F, T)=0$ iff $F$ is constant on $T$. The definition of $\overrightarrow{\mathrm{V}}$ and property (a) of $e$ in Section 2 imply $\overrightarrow{\mathrm{V}}(F, T)=0$ iff $F$ is nondecreasing on $T$ in the sense that $F(s) \subset F(t)$ for all $s, t \in T$ with $s \leq t$. Similarly, $\overleftarrow{\mathrm{V}}(F, T)=0$ iff $F$ is nonincresing on $T$, i.e., $F(s) \supset F(t)$ for all $s, t \in T, s \leq t$.

\subsection{Additivity of Variations}

Since $\left(c(M), d_{H}\right)$ is a metric space, it is known (e.g., [7, 2.19], [8], [9]) that $\mathrm{V}$ is additive (in the second variable) in the sense that

$$
\mathrm{V}(F, T)=\mathrm{V}\left(F, T_{(-\infty, t]}\right)+\mathrm{V}\left(F, T_{[t,+\infty)}\right) \quad \text { for all } t \in T .
$$

We assert that the additivity property (3.1) holds also for $\vec{V}$ and $\overleftarrow{V}$ in place of V. Since the Hausdorff excess $e$ is not symmetric, we have to take care of the order of its arguments. So, we explicitly verify (3.1) at least for $\vec{V}$. 
Proof (of additivity of $\overrightarrow{\mathrm{V}}$ ). Given $\xi, \eta \in \mathbb{R}$ such that $\xi<\overrightarrow{\mathrm{V}}\left(F, T_{(-\infty, t]}\right)$ and $\eta<\overrightarrow{\mathrm{V}}\left(F, T_{[t,+\infty)}\right)$, there are $m, n \in \mathbb{N}$, a partition $\left\{t_{i}\right\}_{i=0}^{m}$ of $T_{(-\infty, t]}$ with $t_{m}=t$ and a partition $\left\{s_{j}\right\}_{j=0}^{n}$ of $T_{[t,+\infty)}$ with $s_{0}=t$ such that

$$
\xi<\sum_{i=1}^{m} e\left(F\left(t_{i-1}\right), F\left(t_{i}\right)\right) \quad \text { and } \quad \eta<\sum_{j=1}^{n} e\left(F\left(s_{j-1}\right), F\left(s_{j}\right)\right) .
$$

Since $\left\{t_{i}\right\}_{i=0}^{m} \cup\left\{s_{j}\right\}_{j=0}^{n}$ is a partition of $T$, we get, from (3.2), $\xi+\eta<\overrightarrow{\mathrm{V}}(F, T)$, which, due to the arbitrariness of $\xi$ and $\eta$ as above, implies the inequality

$$
\overrightarrow{\mathrm{V}}\left(F, T_{(-\infty, t]}\right)+\overrightarrow{\mathrm{V}}\left(F, T_{[t,+\infty)}\right) \leq \overrightarrow{\mathrm{V}}(F, T) .
$$

In order to prove the reverse inequality, let $\xi \in \mathbb{R}$ be arbitrary such that $\xi<\overrightarrow{\mathrm{V}}(F, T)$. Then, there are $m \in \mathbb{N}$ and a partition $\pi=\left\{t_{i}\right\}_{i=0}^{m}$ of $T$ such that the first inequality in (3.2) holds. If $t_{m} \leq t$, then $\pi$ is a partition of $T_{(-\infty, t]}$, and if $t \leq t_{0}$, then $\pi$ is a partition of $T_{[t,+\infty)}$, and so, in the either case, the first inequality in (3.2) implies

$$
\xi<\overrightarrow{\mathrm{V}}\left(F, T_{(-\infty, t]}\right)+\overrightarrow{\mathrm{V}}\left(F, T_{[t,+\infty)}\right) .
$$

Now, suppose $t_{k-1} \leq t \leq t_{k}$ for some $k \in\{1, \ldots, m\}$. By the triangle inequality for $e$, we have

$$
e\left(F\left(t_{k-1}\right), F\left(t_{k}\right)\right) \leq e\left(F\left(t_{k-1}\right), F(t)\right)+e\left(F(t), F\left(t_{k}\right)\right) .
$$

Since $\left\{t_{i}\right\}_{i=0}^{k-1} \cup\{t\}$ is a partition of $T_{(-\infty, t]}$ and $\{t\} \cup\left\{t_{i}\right\}_{i=k}^{m}$ is a partition of $T_{[t,+\infty)}$, from the first inequality in (3.2) and (3.4), once again we get (3.3). By the arbitrariness of $\xi<\overrightarrow{\mathrm{V}}(F, T)$, (3.3) implies

$$
\overrightarrow{\mathrm{V}}(F, T) \leq \overrightarrow{\mathrm{V}}\left(F, T_{(-\infty, t]}\right)+\overrightarrow{\mathrm{V}}\left(F, T_{[t,+\infty)}\right),
$$

which was to be proved.

\subsection{Bounded Directional Variations}

Recall that the function $v_{F}(t):=\mathrm{V}\left(F, T_{(-\infty, t]}\right), t \in T$, is said to be the variation function of $F$ on $T$. We define the variation function of $F$ to the right (to the left) by $\vec{v}_{F}(t):=\overrightarrow{\mathrm{V}}\left(F, T_{(-\infty, t]}\right)$ (by $\overleftarrow{v}_{F}(t):=\overleftarrow{\mathrm{V}}\left(F, T_{(-\infty, t]}\right)$, respectively) for all $t \in T$. Clearly, $v_{F}, \vec{v}_{F}, \overleftarrow{v}_{F}: T \rightarrow[0,+\infty]$ are nondecreasing on $T$. If $F$ is clear from the context, we omit the subscript $F$ and write $v$, $\vec{v}$, and $\overleftarrow{v}$, respectively.

The following characterization holds for multifunctions of bounded directional variation (which will be useful in Section [6). 
Lemma 1. Given $F: T \rightarrow \mathrm{c}(M), \overrightarrow{\mathrm{V}}(F, T)<+\infty$ iff there is a nondecreasing bounded function $\varphi: T \rightarrow \mathbb{R}$ such that $e(F(s), F(t)) \leq \varphi(t)-\varphi(s)$ for all $s, t \in T$ with $s \leq t$; moreover, $\overrightarrow{\mathrm{V}}(F, T) \leq \mathrm{V}(\varphi, T)$. (A similar assertion holds for $\overleftarrow{\mathrm{V}}(F, T)<+\infty$ if the inequality is written as $e(F(t), F(s)) \leq \varphi(t)-\varphi(s)$.)

Proof. $(\Rightarrow)$ Let $\vec{v}(t)=\vec{V}\left(F, T_{(-\infty, t]}\right), t \in T$, be the variation function (to the right) of $F$. It is nondecreasing on $T$ and bounded: $\sup _{t \in T} \vec{v}(t) \leq \overrightarrow{\mathrm{V}}(F, T)$. Given $s, t \in T, s \leq t$, the additivity of $\overrightarrow{\mathrm{V}}$ implies

$$
e(F(s), F(t)) \leq \overrightarrow{\mathrm{V}}\left(F, T_{[s, t]}\right)=\overrightarrow{\mathrm{V}}\left(F, T_{(-\infty, t]}\right)-\overrightarrow{\mathrm{V}}\left(F, T_{(-\infty, s]}\right)=\vec{v}(t)-\vec{v}(s) .
$$

It remains to set $\varphi:=\vec{v}$.

$(\Leftarrow)$ Let $m \in \mathbb{N}$ and $\pi=\left\{t_{i}\right\}_{i=0}^{m}$ be a partition of $T$. Since $t_{i-1} \leq t_{i}$ for all $i=1, \ldots, m$, by the assumption we get

$$
\begin{aligned}
\sum_{i=1}^{m} e\left(F\left(t_{i-1}\right), F\left(t_{i}\right)\right) & \leq \sum_{i=1}^{m}\left(\varphi\left(t_{i}\right)-\varphi\left(t_{i-1}\right)\right)=\varphi\left(t_{m}\right)-\varphi\left(t_{0}\right) \\
& \leq \sup _{t \in T} \varphi(t)-\inf _{s \in T} \varphi(s)=\mathrm{V}(\varphi, T)<+\infty
\end{aligned}
$$

It remains to take the supremum over all partitions $\pi$ of $T$.

Remark 1. It is known $([7,1.23],[12$, Lemma 11]) that if $F: T \rightarrow \mathrm{c}(M)$ and $\mathrm{V}(F, T)<+\infty$, then the image $F(T):=\bigcup_{t \in T} F(t)$ is a totally bounded (hence separable) subset of $M$ (if, in addition, $(M, d)$ is complete, then the closure of $F(T)$ in $M$ is compact). However, condition $\mathrm{V}(F, T)<+\infty$ cannot be replaced neither by $\overrightarrow{\mathrm{V}}(F, T)<+\infty$ nor by $\overleftarrow{\mathrm{V}}(F, T)<+\infty$ (see Example 5 ).

\subsection{Lower Semicontinuity of Variations}

If a sequence $\left\{F_{n}\right\}_{n=1}^{\infty}$ of multifunctions $F_{n}: T \rightarrow \mathrm{c}(M)$ converges in $\mathrm{c}(M)$ pointwise on $T$ to $F$ (i.e., $d_{H}\left(F_{n}(t), F(t)\right) \rightarrow 0$ as $n \rightarrow \infty$ for all $t \in T$ ), then

$$
\mathrm{V}(F, T) \leq \liminf _{n \rightarrow \infty} \mathrm{V}\left(F_{n}, T\right)
$$

This property is known as the (sequential) lower semicontinuity of $\mathrm{V}$ (in the first variable) for metric space valued functions; cf. [9, Proposition 2.1(V7)].

We assert that (3.5) is valid for $\vec{V}$ and $\overleftarrow{V}$ in place of $V$, as well. 
Proof (of lower semicontinuity of $\overrightarrow{\mathrm{V}}$ ). For any $n \in \mathbb{N}$ and $s, t \in T$, the triangle inequality for $e$ implies

$$
\left|e\left(F_{n}(s), F_{n}(t)\right)-e(F(s), F(t))\right| \leq d_{H}\left(F_{n}(s), F(s)\right)+d_{H}\left(F_{n}(t), F(t)\right),
$$

and so, by the pointwise convergence of $F_{n}$ to $F, e\left(F_{n}(s), F_{n}(t)\right) \rightarrow e(F(s), F(t))$ as $n \rightarrow \infty$. Given $m \in \mathbb{N}$ and a partition $\pi=\left\{t_{i}\right\}_{i=0}^{m}$ of $T$, by definition of $\overrightarrow{\mathrm{V}}$,

$$
\sum_{i=1}^{m} e\left(F_{n}\left(t_{i-1}\right), F_{n}\left(t_{i}\right)\right) \leq \overrightarrow{\mathrm{V}}\left(F_{n}, T\right) \quad \text { for all } n \in \mathbb{N} .
$$

Passing to the limit inferior as $n \rightarrow \infty$, we get

$$
\sum_{i=1}^{m} e\left(F\left(t_{i-1}\right), F\left(t_{i}\right)\right) \leq \liminf _{n \rightarrow \infty} \overrightarrow{\mathrm{V}}\left(F_{n}, T\right),
$$

and it remains to take the supremum over all partitions $\pi$ of $T$.

\subsection{Limit Equalities for the Variations}

The following equalities are known $([7,8]$ ) for (multi)functions $F$ on $T$ with values in a metric space (in particular in $\mathrm{c}(M)$ ):

$$
\text { if } s=\sup T \in \mathbb{R} \cup\{+\infty\} \text { and } s \notin T \text {, then } \mathrm{V}(F, T)=\lim _{T \ni t \rightarrow s} \mathrm{~V}\left(F, T_{(-\infty, t]}\right) \text {; }
$$

if $i=\inf T \in \mathbb{R} \cup\{-\infty\}$ and $i \notin T$, then $\mathrm{V}(F, T)=\lim _{T \ni t \rightarrow i} \mathrm{~V}\left(F, T_{[t,+\infty)}\right)$.

We are going to show that these assertions hold for $\vec{V}$ and $\overleftarrow{V}$ as well.

Proof (of (3.6) for $\vec{V}$ ). Since $\vec{v}$ is nondecreasing on $T$, the limit on the right in (3.6) (with $\mathrm{V}$ replaced by $\overrightarrow{\mathrm{V}}$ ) exists in $[0,+\infty]$ and is equal to $\sup _{t \in T} \vec{v}(t)$. Given $t \in T$, we have $T_{(-\infty, t]} \subset T$, and so, $\vec{v}(t) \leq \vec{V}(F, T)$, which implies $\lim _{T \ni t \rightarrow s} \vec{v}(t) \leq \overrightarrow{\mathrm{V}}(F, T)$. Conversely, given $\xi \in \mathbb{R}$ with $\xi<\overrightarrow{\mathrm{V}}(F, T)$, there are $m \in \mathbb{N}$ and a partition $\pi=\left\{t_{i}\right\}_{i=0}^{m}$ of $T$ (and so, $t_{0} \leq t_{1} \leq \cdots \leq t_{m-1} \leq t_{m}<s$ ) such that

$$
\xi<\sum_{i=1}^{m} e\left(F\left(t_{i-1}\right), F\left(t_{i}\right)\right) \leq \vec{v}\left(t_{m}\right) \leq \lim _{T \ni t \rightarrow s} \vec{v}(t) .
$$

Passing to the limit as $\xi \rightarrow \overrightarrow{\mathrm{V}}(F, T)$, we get $\overrightarrow{\mathrm{V}}(F, T) \leq \lim _{T \ni t \rightarrow s} \vec{v}(t)$. 


\subsection{Jump Formulas for the Variations}

We say that $t \in \mathbb{R}$ is a left (right) limit point of $T$ if $T_{(t-\varepsilon, t)} \neq \varnothing$ (respectively, $T_{(t, t+\varepsilon)} \neq \varnothing$ ) for all $\varepsilon>0$. A point $t \in T$, which is not a left (or right) limit point of $T$, is called the left (or right) isolated point of $T$.

By virtue of [9, Lemma 4.2], the following two assertions hold.

(a) If $t \in T$ is a left limit point of $T$ and $\mathrm{V}(F, T)<+\infty$, then

$$
v(t)=v(t-0)+\lim _{T \ni s \rightarrow t-0} d_{H}(F(s), F(t)) \text { 5 }
$$

(b) If $t \in T$ is a right limit point of $T$ and $\mathrm{V}(F, T)<+\infty$, then

$$
v(t+0)=v(t)+\lim _{T \ni s \rightarrow t+0} d_{H}(F(s), F(t)) 6
$$

The second limit in the right-hand side of (3.8) (of (3.9)) is known as the left (right, respectively) jump of $F$ at $t$.

Since $d_{H}$ is symmetric, the order of arguments $F(s)$ and $F(t)$ in (3.8) and (3.9) does not matter. In the counterparts of (a) and (b) for $\vec{V}$ and $\bar{V}$ below, we take care of the order of arguments in the excess $e(\cdot, \cdot)$.

Lemma 2. If $t \in T$ is a left limit point of $T$, then 7

$(\overrightarrow{\mathrm{a}}) \vec{v}(t)=\vec{v}(t-0)+\limsup _{T \ni s \rightarrow t-0} e(F(s), F(t))$ if $\overrightarrow{\mathrm{V}}(F, T)<+\infty$;

$(\overleftarrow{\mathrm{a}}) \overleftarrow{v}(t)=\overleftarrow{v}(t-0)+\limsup _{T \ni s \rightarrow t-0} e(F(t), F(s))$ if $\overleftarrow{\mathrm{V}}(F, T)<+\infty$

If $t \in T$ is a right limit point of $T$, then 8

$(\overrightarrow{\mathrm{b}}) \vec{v}(t+0)=\vec{v}(t)+\limsup _{T \ni s \rightarrow t+0} e(F(t), F(s))$ if $\overrightarrow{\mathrm{V}}(F, T)<+\infty$;

\footnotetext{
${ }^{5} v(t-0):=\lim _{T \ni s \rightarrow t-0} v(s)$ is the left limit of $v$ at $t$ along $T$, i.e., $v(t-0)$ is the limit of $v(s)$ as $s \rightarrow t$ with $s \in T_{(-\infty, t)}$.

${ }^{6} v(t+0):=\lim _{T \ni s \rightarrow t+0} v(s)=\lim _{s \rightarrow t, s \in T_{(t,+\infty)}} v(s)$ is the right limit of $F$ at $t$.

${ }^{7}$ The limit superior in $(\overrightarrow{\mathrm{a}})$ is the limit of $\sup \left\{e(F(s), F(t)): s \in T_{(t-\varepsilon, t)}\right\}$ as $\varepsilon \rightarrow+0$; in $(\bar{a})$ the limit superior is understood similarly.

${ }^{8}$ The limit superior in $(\overrightarrow{\mathrm{b}})$ is the limit of $\sup \left\{e(F(t), F(s)): s \in T_{(t, t+\varepsilon)}\right\}$ as $\varepsilon \rightarrow+0$; the limit superior in $(\overleftarrow{b})$ has a similar meaning.
} 
$(\overleftarrow{\mathrm{b}}) \overleftarrow{v}(t+0)=\overleftarrow{v}(t)+\limsup _{T \ni s \rightarrow t+0} e(F(s), F(t))$ if $\overleftarrow{\mathrm{V}}(F, T)<+\infty$

Proof. We concentrate on $(\vec{a})$ and $(\vec{b})$, making only remarks on $(\overleftarrow{a})$ and $(\overleftarrow{b})$.

$(\overrightarrow{\mathrm{a}})$ First, we prove inequality $\geq$ in $(\overrightarrow{\mathrm{a}})$. By the additivity of $\vec{V}$, given $s \in T$ with $s<t$, we have

$$
\vec{v}(s)+e(F(s), F(t)) \leq \vec{v}(s)+\vec{V}\left(F, T_{[s, t]}\right)=\vec{v}(t) .
$$

Since $\vec{v}$ is nondecreasing and bounded on $T$ (by $\vec{V}(F, T)$ ), and $t$ is a left limit point of $T$, the left limit $\vec{v}(t-0)$ exists in $[0,+\infty)$ and is equal to $\sup \{\vec{v}(s): s \in T, s<t\}$. Inequality $\geq$ in $(\overrightarrow{\mathrm{a}})$ follows now from the properties of the limit superior as $T \ni s \rightarrow t-0$, since the right-hand side in $(\overrightarrow{\mathrm{a}})$ is equal to

$$
\lim _{T \ni s \rightarrow t-0} \vec{v}(s)+\limsup _{T \ni s \rightarrow t-0} e(F(s), F(t))=\limsup _{T \ni s \rightarrow t-0}(\vec{v}(s)+e(F(s), F(t))) \leq \vec{v}(t) .
$$

Now we show that inequality $\leq$ holds in $(\overrightarrow{\mathrm{a}})$. By the definition of $\vec{v}(t)$, which is finite, for any $\xi \in \mathbb{R}, \xi<\vec{v}(t)$, there are $m \in \mathbb{N}$ and a partition $\pi=\left\{t_{i}\right\}_{i=0}^{m} \cup\{t\}$ of $T_{(-\infty, t]}$ with $t_{m}<t$ such that

$$
\xi<\sum_{i=1}^{m} e\left(F\left(t_{i-1}\right), F\left(t_{i}\right)\right)+e\left(F\left(t_{m}\right), F(t)\right) .
$$

Given $s \in T_{\left(t_{m}, t\right)}$, the triangle inequality for $e$ implies

$$
e\left(F\left(t_{m}\right), F(t)\right) \leq e\left(F\left(t_{m}\right), F(s)\right)+e(F(s), F(t)),
$$

and so, $\xi<\vec{v}(s)+e(F(s), F(t))$. Passing to the limit superior as $T \ni s \rightarrow$ $t-0$, we get

$$
\xi \leq \vec{v}(t-0)+\limsup _{T \ni s \rightarrow t-0} e(F(s), F(t)) .
$$

It remains to take into account the arbitrariness of $\xi<\vec{v}(t)$.

$(\vec{b})$ In order to prove inequality $\geq$ in $(\vec{b})$, we make use of the additivity of $\vec{V}$ to get

$$
\vec{v}(t)+e(F(t), F(s)) \leq \vec{v}(t)+\overrightarrow{\mathrm{V}}\left(F, T_{[t, s]}\right)=\vec{v}(s) \quad \text { for all } s \in T_{(t,+\infty)} .
$$

Now, it suffices to pass to the limit superior as $T \ni s \rightarrow t+0$. 
To show inequality $\leq$ in $(\vec{b})$, we apply the additivity property of $\vec{V}$ several times. To begin with, note that

$$
\overrightarrow{\mathrm{V}}\left(F, T_{[t,+\infty)}\right)=\overrightarrow{\mathrm{V}}(F, T)-\vec{v}(t) .
$$

Given $\xi \in \mathbb{R}$ with $\xi<\overrightarrow{\mathrm{V}}\left(F, T_{[t,+\infty)}\right)$, there exist $m \in \mathbb{N}$ and a partition $\{t\} \cup\left\{t_{i}\right\}_{i=0}^{m}$ of $T_{[t,+\infty)}$ with $t<t_{0}$ such that

$$
\xi<e\left(F(t), F\left(t_{0}\right)\right)+\sum_{i=1}^{m} e\left(F\left(t_{i-1}\right), F\left(t_{i}\right)\right) .
$$

Since, for any $s \in T_{\left(t, t_{0}\right)}, e\left(F(t), F\left(t_{0}\right)\right) \leq e(F(t), F(s))+e\left(F(s), F\left(t_{0}\right)\right)$, we find

$$
\begin{aligned}
\xi+\vec{v}\left(t_{m}\right)-\overrightarrow{\mathrm{V}}(F, T) \leq \xi & <e(F(t), F(s))+\overrightarrow{\mathrm{V}}\left(F, T_{\left[s, t_{m}\right]}\right) \\
& =e(F(t), F(s))+\vec{v}\left(t_{m}\right)-\vec{v}(s),
\end{aligned}
$$

and so, $\vec{v}(s) \leq(\overrightarrow{\mathrm{V}}(F, T)-\xi)+e(F(t), F(s))$. Passing to the limit superior as $T \ni s \rightarrow t+0$, we get

$$
\vec{v}(t+0) \leq \overrightarrow{\mathrm{V}}(F, T)-\xi+\limsup _{T \ni s \rightarrow t+0} e(F(t), F(s)) .
$$

It remains to let $\xi$ tend to the value (3.10).

$(\overleftarrow{\mathrm{a}}),(\overleftarrow{\mathrm{b}})$ Here we note only that, by the additivity of $\overleftarrow{\mathrm{V}}$,

$$
\begin{array}{ll}
\overleftarrow{v}(s)+e(F(t), F(s)) \leq \overleftarrow{v}(s)+\overleftarrow{V}\left(F, T_{[s, t]}\right)=\overleftarrow{v}(t) & \forall s \in T_{(-\infty, t)} \\
\overleftarrow{v}(t)+e(F(s), F(t)) \leq \overleftarrow{v}(t)+\overleftarrow{V}\left(F, T_{[t, s]}\right)=\overleftarrow{v}(s) & \forall s \in T_{(t,+\infty)}
\end{array}
$$

respectively.

As a corollary of (3.6), (3.7), and Lemma 2, we get the following lemma, which is a generalization of Theorem 4.6 from [9].

Lemma 3. If $t \in T$ is a left limit point of $T$, then

(a) $\overrightarrow{\mathrm{V}}\left(F, T_{(-\infty, t]}\right)=\overrightarrow{\mathrm{V}}\left(F, T_{(-\infty, t)}\right)+\limsup _{T \ni s \rightarrow t-0} e(F(s), F(t))$ if $\overrightarrow{\mathrm{V}}(F, T)<+\infty$;

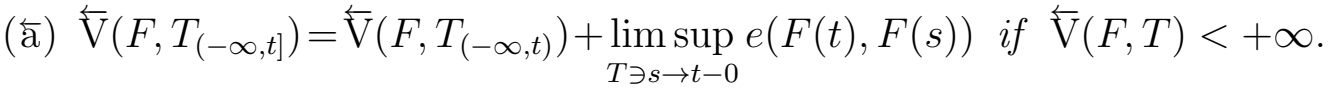


If $t \in T$ is a right limit point of $T$, then

$(\overrightarrow{\mathrm{b}}) \overrightarrow{\mathrm{V}}\left(F, T_{[t,+\infty)}\right)=\overrightarrow{\mathrm{V}}\left(F, T_{(t,+\infty)}\right)+\limsup _{T \ni s \rightarrow t+0} e(F(t), F(s))$ if $\overrightarrow{\mathrm{V}}(F, T)<+\infty$;

$(\overleftarrow{\mathrm{b}}) \overleftarrow{\mathrm{V}}\left(F, T_{[t,+\infty)}\right)=\overleftarrow{\mathrm{V}}\left(F, T_{(t,+\infty)}\right)+\limsup _{T \ni s \rightarrow t+0} e(F(s), F(t))$ if $\overleftarrow{\mathrm{V}}(F, T)<+\infty$

Proof. As in the proof of Lemma 2 , we concentrate on $(\overrightarrow{\mathrm{a}})$ and $(\overrightarrow{\mathrm{b}})$.

$(\vec{a})$ This is a consequence of Lemma $2(\vec{a})$, since $\vec{V}\left(F, T_{(-\infty, t]}\right)=\vec{v}(t)$ and, by virtue of (3.6) $), \overrightarrow{\mathrm{V}}\left(F, T_{(-\infty, t)}\right)=\vec{v}(t-0)$.

$(\overrightarrow{\mathrm{b}})$ For every $s \in T_{(t,+\infty)}$, the additivity of $\overrightarrow{\mathrm{V}}$ implies

$$
\overrightarrow{\mathrm{V}}\left(F, T_{[t,+\infty)}\right)=\vec{v}(s)-\vec{v}(t)+\overrightarrow{\mathrm{V}}\left(F, T_{[s,+\infty)}\right) .
$$

Passing to the limit as $T \ni s \rightarrow t+0$, we get: by Lemma $2(\overrightarrow{\mathrm{b}})$,

$$
\vec{v}(s)-\vec{v}(t) \rightarrow \vec{v}(t+0)-\vec{v}(t)=\limsup _{T \ni s \rightarrow t+0} e(F(t), F(s)),
$$

and by (3.7), $\overrightarrow{\mathrm{V}}\left(F, T_{[s,+\infty)}\right) \rightarrow \overrightarrow{\mathrm{V}}\left(F, T_{(t,+\infty)}\right)$.

\subsection{Pointwise Selection Principle}

In the proof of our main result (Theorem 1), we will need a compactness theorem in the topology of pointwise convergence (cf. Section 3.4) for a (approximating) sequence of multifunctions $F_{n}: T \rightarrow \mathrm{c}(M), n \in \mathbb{N}$, reformulated from [14] for the metric space $\left(\mathrm{c}(M), d_{H}\right)$ under consideration.

Given $F: T \rightarrow \mathrm{c}(M)$, its modulus of variation is the nondecreasing sequence $\left\{\nu_{k}(F, T)\right\}_{k=1}^{\infty} \subset[0,+\infty]$ defined by

$$
\nu_{k}(F, T):=\sup \sum_{i=1}^{k} d_{H}\left(F\left(s_{i}\right), F\left(t_{i}\right)\right),
$$

the supremum being taken over all collections $s_{1}, \ldots, s_{k}, t_{1} \ldots, t_{k}$ of $2 k$ numbers from $T$ such that $s_{1} \leq t_{1} \leq s_{2} \leq t_{2} \leq \cdots \leq s_{k-1} \leq t_{k-1} \leq s_{k} \leq t_{k}$. This notion was introduced in [6] in the context of Fourier series and extensively applied in [21, Section 11.3] for real valued functions. The general case of metric space valued functions was considered in [14], whence we know that $\nu_{k}(F, T) \leq \mathrm{V}(F, T)$ for all $k \in \mathbb{N}$, and $\nu_{k}(F, T) \rightarrow \mathrm{V}(F, T)$ as $k \rightarrow \infty$.

The following theorem, extending Helly's Selection Theorem, is a pointwise selection principle in terms of the modulus of variation: 
Theorem B ([14, Theorem 1]). Suppose $\left\{F_{n}\right\}_{n=1}^{\infty}$ is a sequence of multifunctions $F_{n}: T \rightarrow \mathrm{c}(M)$ such that (a) $\limsup _{n \rightarrow \infty} \nu_{k}\left(F_{n}, T\right)=o(k) 9$, and (b) the closure in $\mathrm{c}(M)$ of the set $\left\{F_{n}(t): n \in \mathbb{N}\right\}$ is compact for all $t \in T$. Then $\left\{F_{n}\right\}_{n=1}^{\infty}$ admits a subsequence, which converges in $\mathrm{c}(M)$ pointwise on $T$ to a multifunction $F: T \rightarrow \mathrm{c}(M)$ such that $\nu_{k}(F, T)=o(k)$.

\section{Proof of the Main Result}

Proof of Theorem 1. We prove only item (a), item (b) being proved similarly with corresponding modifications ('to the left'). We divide the proof into six steps for clarity. Recall that $a=\inf T \in T$, and we set $b:=\sup T$. In the first four steps, we prove the theorem in the case when $t_{0}=a$ and $b \in T$ (so that $T \subset\left[t_{0}, b\right]$ is bounded), in step 5 - when $t_{0}=a$ and $b \notin T$, and in step 6 - when $t_{0}>a$ and $T$ is arbitrary. We employ several ideas from [3, 9], [13, Sections 5, 10].

Step 1. Suppose $T \subset\left[t_{0}, b\right]$ and $t_{0}, b \in T$. Since $\overrightarrow{\mathrm{V}}(F, T)$ is finite and the variation function of $F$ to the right $\vec{v}: T \rightarrow[0,+\infty)$ is nondecreasing on $T$, the set of its points of discontinuity on $T$ is at most countable. Denote by $T_{F}$ the set of points $t \in T$, which are left limit points of $T$ such that $\vec{v}(t)=$ $\vec{v}(t-0)$. It follows that $T \backslash T_{F}$ is at most countable and, by Lemma $2(\vec{a})$,

$$
\text { if } t \in T_{F} \text {, then } e(F(s), F(t)) \rightarrow 0 \text { as } T \ni s \rightarrow t-0 \text {. }
$$

Furthermore, the set $T_{0}$ of left isolated points of $T$ (i.e., $t \in T$ such that $T_{(t-\varepsilon, t)}=\varnothing$ for some $\varepsilon>0$ ) is also at most countable (in fact, the intervals of "emptiness from the left", corresponding to different left isolated points of $T$, are disjoint, and each such interval contains a rational number). Let $Q$ denote an at most countable dense subset of $T$. We set

$$
S:=\left\{t_{0}, b\right\} \cup\left(T \backslash T_{F}\right) \cup T_{0} \cup Q
$$

and note that $S \subset T$ is dense in $T$ and at most countable. With no loss of generality we may assume that $S$ is countable, say, $S=\left\{t_{i}\right\}_{i=0}^{\infty}$. Given $n \in \mathbb{N}$, the set $\pi_{n}=\left\{t_{i}\right\}_{i=0}^{n-1} \cup\{b\}$ is a partition of $T$. Ordering the points in $\pi_{n}$ in ascending order and denoting the resulting (ordered) partition of $T$ by

\footnotetext{
${ }^{9}$ Equality $\mu_{k}=o(k)$ means as usual that $\lim _{k \rightarrow \infty} \mu_{k} / k=0$.
} 
$\pi_{n}=\left\{t_{i}^{n}\right\}_{i=0}^{n}$, we get

$$
\begin{gathered}
t_{0}=t_{0}^{n}<t_{1}^{n}<\cdots<t_{n-1}^{n}<t_{n}^{n}=b, \text { and } \\
\forall t \in S \exists n_{0}=n_{0}(t) \in \mathbb{N} \text { such that } t \in \pi_{n} \text { for all } n \geq n_{0} .
\end{gathered}
$$

Step 2. Let us construct an approximating sequence for the desired setvalued selector of $F$. In order to do this, we need the following observation from [13, assertion (10.2)]: given $X, Y \in \mathrm{c}(M)$,

there is $Y^{\prime} \in \mathrm{c}(M)$ such that $Y^{\prime} \subset Y$ and $d_{H}\left(X, Y^{\prime}\right) \leq e(X, Y)$.

In fact, it suffices to define $Y^{\prime}$ as the metric projection of $X$ onto $Y$ :

$$
Y^{\prime}=\operatorname{Pr}_{Y} X:=\{y \in Y \text { : there is } x \in X \text { such that } d(x, y)=d(x, Y)\} .
$$

First, given $n \in \mathbb{N}$, we define sets $Y_{i}^{n} \in \mathrm{c}(M), i=0,1, \ldots, n$, inductively as follows. Setting $X=X_{0}$ and $Y=F\left(t_{0}\right)$ in (4.4), we choose $Y_{0}:=Y^{\prime} \in$ $\mathrm{c}(M)$ such that $Y_{0} \subset F\left(t_{0}\right)$ and $d_{H}\left(X_{0}, Y_{0}\right) \leq e\left(X_{0}, F\left(t_{0}\right)\right)$. We put $Y_{0}^{n}:=Y_{0}$ (for all $n \in \mathbb{N}$ ). Now, suppose $i \in\{1, \ldots, n\}$ and the set $Y_{i-1}^{n} \in \mathrm{c}(M)$ such that $Y_{i-1}^{n} \subset F\left(t_{i-1}^{n}\right)$ is already chosen. Then, we put $X=Y_{i-1}^{n}$ and $Y=F\left(t_{i}^{n}\right)$ in (4.4) and pick $Y_{i}^{n}:=Y^{\prime} \in \mathrm{c}(M)$ such that $Y_{i}^{n} \subset F\left(t_{i}^{n}\right)$ and

$$
d_{H}\left(Y_{i-1}^{n}, Y_{i}^{n}\right) \leq e\left(Y_{i-1}^{n}, F\left(t_{i}^{n}\right)\right) \leq e\left(F\left(t_{i-1}^{n}\right), F\left(t_{i}^{n}\right)\right) .
$$

The approximating sequence $\Gamma_{n}: T \rightarrow \mathrm{c}(M), n \in \mathbb{N}$, is defined as a sequence of set-valued step functions of the form (cf. [13, equation (10.3)]):

$$
\begin{aligned}
\Gamma_{n}\left(t_{i}^{n}\right) & :=Y_{i}^{n} \quad \text { for all } i=0,1, \ldots, n \\
\Gamma_{n}(t) & :=Y_{i-1}^{n} \text { for all } t \in T \cap\left(t_{i-1}^{n}, t_{i}^{n}\right) \text { and } i=1, \ldots, n
\end{aligned}
$$

(if $T \cap\left(t_{i-1}^{n}, t_{i}^{n}\right)=\varnothing$, then $\Gamma_{n}$ is left undefined on $\left.\left(t_{i-1}^{n}, t_{i}^{n}\right)\right)$. Clearly,

$$
\Gamma_{n}\left(t_{0}\right)=\Gamma_{n}\left(t_{0}^{n}\right)=Y_{0}^{n}=Y_{0} \subset F\left(t_{0}\right) \text { for all } n \in \mathbb{N} .
$$

Moreover, by the additivity of $\mathrm{V}$ and (4.6), we have

$$
\begin{aligned}
\mathrm{V}\left(\Gamma_{n}, T\right) & =\sum_{i=1}^{n} \mathrm{~V}\left(\Gamma_{n}, T \cap\left[t_{i-1}^{n}, t_{i}^{n}\right]\right)=\sum_{i=1}^{n} d_{H}\left(Y_{i-1}^{n}, Y_{i}^{n}\right) \\
& \leq \sum_{i=1}^{n} e\left(F\left(t_{i-1}^{n}\right), F\left(t_{i}^{n}\right)\right) \leq \overrightarrow{\mathrm{V}}(F, T) \text { for all } n \in \mathbb{N} .
\end{aligned}
$$


Step 3. Let us show that $\left\{\Gamma_{n}\right\}_{n=1}^{\infty}$ satisfies the assumptions of Theorem B. By virtue of (4.9), we get

$$
\limsup _{n \rightarrow \infty} \nu_{k}\left(\Gamma_{n}, T\right) \leq \overrightarrow{\mathrm{V}}(F, T) \text { for all } k \in \mathbb{N}
$$

and so, condition (a) in Theorem $\mathrm{B}$ is satisfied. Now, we verify condition (b).

If $t \in S$, then, by (4.3), there is $n_{0}=n_{0}(t) \in \mathbb{N}$ such that $t \in \pi_{n}$ for all $n \geq n_{0}$. So, for each $n \geq n_{0}$ there is $i=i(t, n) \in\{0,1, \ldots, n\}$ such that $t=t_{i}^{n}$. The definition of $\Gamma_{n}$ implies

$$
\Gamma_{n}(t)=\Gamma_{n}\left(t_{i}^{n}\right)=Y_{i}^{n} \subset F\left(t_{i}^{n}\right)=F(t) \text { for all } n \geq n_{0} .
$$

In other words, $\left\{\Gamma_{n}(t)\right\}_{n=n_{0}}^{\infty} \subset \mathrm{c}(F(t))$. Since $F(t)$ is a compact subset of $M$, it follows from [5, II.1.4] that $\mathrm{c}(F(t))$ is a compact subset of $\mathrm{c}(M)$, which implies the desired property for $\left\{F_{n}(t)\right\}_{n=1}^{\infty}$.

Suppose now that $t \in T \backslash S$. We have $t \in T_{F} \cap\left(t_{0}, b\right)$, i.e., by (4.1),

$$
e(F(s), F(t)) \rightarrow 0 \quad \text { as } \quad T_{\left(t_{0}, b\right)} \ni s \rightarrow t-0,
$$

where $t$ is a left limit point of $T$, and so, there is a sequence $\tau_{k} \in T, \tau_{k}<t$, $k \in \mathbb{N}$, such that $\tau_{k} \rightarrow t$ as $k \rightarrow \infty$. Since $S$ is dense in $T$, given $k \in \mathbb{N}$, there is $s_{k} \in S$ such that $\left|s_{k}-\tau_{k}\right|<t-\tau_{k}$, which implies $s_{k}<t$, and $s_{k} \rightarrow t$ as $k \rightarrow \infty$. From (4.3), for each $k \in \mathbb{N}$ choose a number $n_{0}(k) \in \mathbb{N}$ (depending also on $t$ ) such that $s_{k} \in \pi_{n}$ for all $n \geq n_{0}(k)$. We may assume (arguing inductively) that the sequence $\left\{n_{0}(k)\right\}_{k=1}^{\infty}$ is strictly increasing. Given $k \in \mathbb{N}$ and $n \geq$ $n_{0}(k)$, since $s_{k} \in \pi_{n}$, there is a number $j(k, n) \in\{0,1, \ldots, n-1\}$ such that $s_{k}=t_{j(k, n)}^{n}$ and, since $s_{k}<t$, there is (unique) $i(k, n) \in\{j(k, n), \ldots, n-1\}$ such that

$$
s_{k}=t_{j(k, n)}^{n} \leq t_{i(k, n)}^{n}<t<t_{i(k, n)+1}^{n} .
$$

By (4.7), (4.8), and (4.12), we find

$$
\Gamma_{n}(t)=Y_{i(k, n)}^{n} \subset F\left(t_{i(k, n)}^{n}\right) \quad \text { for all } k \in \mathbb{N} \text { and } n \geq n_{0}(k) .
$$

Setting $n:=n_{0}(k)$ and $p_{k}:=t_{i\left(k, n_{0}(k)\right)}^{n_{0}(k)}$ in (4.13), we have $\Gamma_{n_{0}(k)}(t) \subset F\left(p_{k}\right)$ for all $k \in \mathbb{N}$, where, by virtue of (4.12) and property $s_{k} \rightarrow t$ as $k \rightarrow \infty$,

$$
p_{k}<t \text { and } p_{k} \rightarrow t \text { as } k \rightarrow \infty \text {. }
$$

Applying (4.4), for each $k \in \mathbb{N}$ pick $Y_{k}(t) \in \mathrm{c}(M)$ such that $Y_{k}(t) \subset F(t)$ and

$$
d_{H}\left(\Gamma_{n_{0}(k)}, Y_{k}(t)\right) \leq e\left(\Gamma_{n_{0}(k)}, F(t)\right) \leq e\left(F\left(p_{k}\right), F(t)\right) .
$$


It follows from (4.11) and (4.14) that the right-hand side in (4.15) tends to zero as $k \rightarrow \infty$. By the compactness of $\mathrm{c}(F(t))$, we may assume (passing to a subsequence of $\left\{Y_{k}(t)\right\}_{k=1}^{\infty}$ if necessary) that $d_{H}\left(Y_{k}(t), Y(t)\right) \rightarrow 0$ as $k \rightarrow \infty$ for some $Y(t) \in \mathrm{c}(M)$ such that $Y(t) \subset F(t)$. Thus,

$$
d_{H}\left(\Gamma_{n_{0}(k)}(t), Y(t)\right) \leq d_{H}\left(\Gamma_{n_{0}(k)}(t), Y_{k}(t)\right)+d_{H}\left(Y_{k}(t), Y(t)\right) \rightarrow 0 \quad \text { as } k \rightarrow \infty,
$$

and so, (a subsequence of) the subsequence $\left\{\Gamma_{n_{0}(k)}\right\}_{k=1}^{\infty}$ of $\left\{\Gamma_{n}(t)\right\}_{n=1}^{\infty}$ converges in $\mathrm{c}(M)$. This finishes the proof of compactness of $\overline{\left\{\Gamma_{n}(t)\right\}_{n=1}^{\infty}}$ (the closure being taken in $\mathrm{c}(M))$.

Step 4. By Theorem B, there are a subsequence of $\left\{\Gamma_{n}\right\}_{n=1}^{\infty}$, which we denote by $\left\{\Gamma_{l(n)}\right\}_{n=1}^{\infty}$ with strictly increasing $l: \mathbb{N} \rightarrow \mathbb{N}$, and a multifunction $\Gamma: T \rightarrow \mathrm{c}(M)$ such that $d_{H}\left(\Gamma_{l(n)}(t), \Gamma(t)\right) \rightarrow 0$ as $n \rightarrow \infty$ for all $t \in T$.

Let us show that $\Gamma$ is a set-valued selector of $F$ on $T$. It is clear from (4.7) and (4.2) that $\Gamma\left(t_{0}\right)=Y_{0} \subset F\left(t_{0}\right)$, and so (cf. Step 2),

$$
d_{H}\left(X_{0}, \Gamma\left(t_{0}\right)\right) \leq e\left(X_{0}, F\left(t_{0}\right)\right) .
$$

If $t \in S$, (4.3) implies $t \in \pi_{n}$ for some $n_{0}=n_{0}(t) \in \mathbb{N}$ and all $n \geq n_{0}$. Since $l(n) \geq l\left(n_{0}\right) \geq n_{0}$ for $n \geq n_{0}$, it follows from (4.10) that $\Gamma_{l(n)}(t) \subset F(t)$, and so (cf. properties (a) and (b) of $e$ in Section 2),

$$
\begin{aligned}
e(\Gamma(t), F(t)) & \leq e\left(\Gamma(t), \Gamma_{l(n)}(t)\right)+e\left(\Gamma_{l(n)}(t), F(t)\right) \\
& =e\left(\Gamma(t), \Gamma_{l(n)}(t)\right) \leq d_{H}\left(\Gamma(t), \Gamma_{l(n)}(t)\right) \rightarrow 0 \quad \text { as } n \rightarrow \infty .
\end{aligned}
$$

This gives $e(\Gamma(t), F(t))=0$ implying $\Gamma(t) \subset F(t)$.

Now suppose that $t \in T \backslash S$. Given $k \in \mathbb{N}$, let $n_{0}(k)$ be the number (also depending on $t$ ) from Step 3 such that $s_{k} \in \pi_{n}$ for all $n \geq n_{0}(k)$. Hence, assertions (4.12) and (4.13) still hold. Setting $n=N(k):=l\left(n_{0}(k)\right) \geq n_{0}(k)$ and $q_{k}:=t_{i(k, N(k))}^{N(k)}$ in (4.12) and (4.13), we find $\Gamma_{N(k)} \subset F\left(q_{k}\right)$, where $q_{k}<t$, and $q_{k} \rightarrow t$ as $k \rightarrow \infty$. For each $k \in \mathbb{N}$, thanks to (4.4), let $Z_{k}(t) \in \mathrm{c}(M)$ be such that $Z_{k}(t) \subset F(t)$ and

$$
d_{H}\left(\Gamma_{N(k)}(t), Z_{k}(t)\right) \leq e\left(\Gamma_{N(k)}(t), F(t)\right) \leq e\left(F\left(q_{k}\right), F(t)\right) .
$$

Noting that $e\left(Z_{k}(t), F(t)\right)=0$, we get

$$
e(\Gamma(t), F(t)) \leq e\left(\Gamma(t), \Gamma_{N(k)}(t)\right)+e\left(\Gamma_{N(k)}(t), Z_{k}(t)\right)+e\left(Z_{k}(t), F(t)\right)
$$


with the right-hand side tending to zero as $n \rightarrow \infty$. Hence $e(\Gamma(t), F(t))=0$, and so, $\Gamma(t) \subset F(t)$. Thus, we have shown that $\Gamma(t) \subset F(t)$ for all $t \in T$.

The lower semicontinuity of $\mathrm{V}$ and (4.9) imply

$$
\mathrm{V}(\Gamma, T) \leq \liminf _{n \rightarrow \infty} \mathrm{V}\left(\Gamma_{l(n)}, T\right) \leq \overrightarrow{\mathrm{V}}(F, T) .
$$

Since $T_{\left[a, t_{0}\right)}=\varnothing\left(\right.$ recall that $\left.t_{0}=a\right), T_{\left[t_{0},+\infty\right)}=T_{\left[t_{0}, b\right]}=T$, and $J_{a}\left(\Gamma, t_{0}\right)=0$, this proves Theorem 1(a) in the case when $t_{0}=a$ and $b=\sup T \in T$.

Remark. Note that if $X_{0} \subset F\left(t_{0}\right)$, we have, by (4.16), $\Gamma\left(t_{0}\right)=X_{0}$.

Step 5. Suppose $t_{0}=a$ and $b=\sup T \notin T$, so that $T_{\left[a, t_{0}\right)}=\varnothing, T_{\left[t_{0},+\infty\right)}=T$, and $J_{a}\left(\Gamma, t_{0}\right)=0$. Pick an increasing sequence $\left\{t_{k}\right\}_{k=1}^{\infty} \subset T$ such that $t_{k} \rightarrow b$ as $k \rightarrow \infty$. Noting that $\overrightarrow{\mathrm{V}}\left(F, T_{\left[t_{0}, t_{1}\right]}\right) \leq \overrightarrow{\mathrm{V}}(F, T)<+\infty$ and applying Steps $1-4$ to $F$ on $T_{\left[t_{0}, t_{1}\right]}$, we get a set-valued selector $\Gamma_{0}: T_{\left[t_{0}, t_{1}\right]} \rightarrow \mathrm{c}(M)$ of $F$ on $T_{\left[t_{0}, t_{1}\right]}$ such that

$$
d_{H}\left(X_{0}, \Gamma_{0}\left(t_{0}\right)\right) \leq e\left(X_{0}, F\left(t_{0}\right)\right) \quad \text { and } \quad \mathrm{V}\left(\Gamma_{0}, T_{\left[t_{0}, t_{1}\right]}\right) \leq \overrightarrow{\mathrm{V}}\left(F, T_{\left[t_{0}, t_{1}\right]}\right) .
$$

Inductively, if $k \in \mathbb{N}$, and a set-valued selector $\Gamma_{k-1}$ of $F$ on $T_{\left[t_{k-1}, t_{k}\right]}$ is already chosen, we note that $\vec{V}\left(F, T_{\left[t_{k}, t_{k+1}\right]}\right) \leq \vec{V}(F, T)<+\infty$, again apply Steps $1-4$, and find a set-valued selector $\Gamma_{k}$ of $F$ on $T_{\left[t_{k}, t_{k+1}\right]}$ such that $\Gamma_{k}\left(t_{k}\right)=\Gamma_{k-1}\left(t_{k}\right)$ and $\mathrm{V}\left(\Gamma_{k}, T_{\left[t_{k}, t_{k+1}\right]}\right) \leq \overrightarrow{\mathrm{V}}\left(F, T_{\left[t_{k}, t_{k+1}\right]}\right)$. Since $t_{k} \rightarrow b$ as $k \rightarrow \infty$, and $b \notin T$, we have $T=\bigcup_{k=0}^{\infty} T_{\left[t_{k}, t_{k+1}\right]}$, so if $t \in T$ and $t \in T_{\left[t_{k}, t_{k+1}\right]}$ for some $k \in\{0\} \cup \mathbb{N}$, we set $\Gamma(t):=\Gamma_{k}(t)$. Clearly, $\Gamma: T \rightarrow \mathrm{c}(M)$ is a well-defined set-valued selector of $F$ on $T$, inequality (4.16) holds, and, by (3.6) and the additivity of $\mathrm{V}$ and $\overrightarrow{\mathrm{V}}$,

$$
\begin{aligned}
\mathrm{V}(\Gamma, T) & =\lim _{n \rightarrow \infty} \mathrm{V}\left(\Gamma, T_{\left(-\infty, t_{n}\right]}\right)=\lim _{n \rightarrow \infty} \sum_{k=0}^{n-1} \mathrm{~V}\left(\Gamma_{k}, T_{\left[t_{k}, t_{k+1}\right]}\right) \\
& \leq \lim _{n \rightarrow \infty} \sum_{k=0}^{n-1} \overrightarrow{\mathrm{V}}\left(F, T_{\left[t_{k}, t_{k+1}\right]}\right)=\lim _{n \rightarrow \infty} \overrightarrow{\mathrm{V}}\left(F, T_{\left(-\infty, t_{n}\right]}\right)=\overrightarrow{\mathrm{V}}(F, T) .
\end{aligned}
$$

Step 6. Finally, suppose $t_{0}>a$. Since $\vec{V}\left(F, T_{\left[a, t_{0}\right)}\right)$ and $\vec{V}\left(F, T_{\left[t_{0},+\infty\right)}\right)$ do not exceed $\overrightarrow{\mathrm{V}}(F, T)<+\infty$, and $X_{0} \in \mathrm{c}(M)$, we apply Steps $1-5$ twice: first, to $F$ on $T_{\left[a, t_{0}\right)}$ with arbitrary $K_{0} \in \mathrm{c}(M)$ in order to obtain a set-valued selector $\Gamma_{1}$ of $F$ on $T_{\left[a, t_{0}\right)}$ such that

$$
d_{H}\left(K_{0}, \Gamma_{1}(a)\right) \leq e\left(K_{0}, F(a)\right) \quad \text { and } \quad \mathrm{V}\left(\Gamma_{1}, T_{\left[a, t_{0}\right)}\right) \leq \overrightarrow{\mathrm{V}}\left(F, T_{\left[a, t_{0}\right)}\right),
$$


and, second, to $F$ on $T_{\left[t_{0},+\infty\right)}$ in order to obtain a set-valued selector $\Gamma_{2}$ of $F$ on $T_{\left[t_{0},+\infty\right)}$ such that

$$
d_{H}\left(X_{0}, \Gamma_{2}\left(t_{0}\right)\right) \leq e\left(X_{0}, F\left(t_{0}\right)\right) \quad \text { and } \quad \mathrm{V}\left(\Gamma_{2}, T_{\left[t_{0},+\infty\right)}\right) \leq \overrightarrow{\mathrm{V}}\left(F, T_{\left[t_{0},+\infty\right)}\right) .
$$

Given $t \in T$, we set $\Gamma(t):=\Gamma_{1}(t)$ if $t \in T_{\left[a, t_{0}\right)}$, and $\Gamma(t):=\Gamma_{2}(t)$ if $t \in T_{\left[t_{0},+\infty\right)}$. Clearly, $\Gamma: T \rightarrow \mathrm{c}(M)$ is a set-valued selector of $F$ on $T$, inequality (4.16) holds, $\mathrm{V}\left(\Gamma, T_{\left[a, t_{0}\right)}\right) \leq \overrightarrow{\mathrm{V}}\left(F, T_{\left[a, t_{0}\right)}\right)$, and $\mathrm{V}\left(\Gamma, T_{\left[t_{0},+\infty\right)}\right) \leq \overrightarrow{\mathrm{V}}\left(F, T_{\left[t_{0},+\infty\right)}\right)$. Furthermore, since $\overrightarrow{\mathrm{V}}$ is additive, the second inequality in (2.1) holds (recall that $a=\min T)$ :

$$
\begin{aligned}
\overrightarrow{\mathrm{V}}\left(F, T_{\left[a, t_{0}\right)}\right)+\overrightarrow{\mathrm{V}}\left(F, T_{\left[t_{0},+\infty\right)}\right) & \leq \overrightarrow{\mathrm{V}}\left(F, T_{\left[a, t_{0}\right]}\right)+\overrightarrow{\mathrm{V}}\left(F, T_{\left[t_{0},+\infty\right)}\right)= \\
& =\overrightarrow{\mathrm{V}}\left(F, T_{[a,+\infty)}\right)=\overrightarrow{\mathrm{V}}(F, T) .
\end{aligned}
$$

Let us prove the first inequality in (2.1).

Suppose $s_{0} \in T_{\left[a, t_{0}\right)}$. Hence $s_{0}=\max T_{\left[a, t_{0}\right)}<t_{0}$, and so, $T_{\left[a, t_{0}\right)}=T_{\left[a, s_{0}\right]}$ and $T_{\left[s_{0}, t_{0}\right]}=\left\{s_{0}, t_{0}\right\}$ (two-point set). By the additivity of $\mathrm{V}$,

$$
\begin{aligned}
\mathrm{V}\left(\Gamma, T_{\left[a, t_{0}\right]}\right) & =\mathrm{V}\left(\Gamma, T_{\left[a, s_{0}\right]}\right)+\mathrm{V}\left(\Gamma, T_{\left[s_{0}, t_{0}\right]}\right) \\
& =\mathrm{V}\left(\Gamma_{1}, T_{\left[a, t_{0}\right)}\right)+d_{H}\left(\Gamma_{1}\left(s_{0}\right), \Gamma_{2}\left(t_{0}\right)\right),
\end{aligned}
$$

which implies

$$
\begin{aligned}
\mathrm{V}(\Gamma, T) & =\mathrm{V}\left(\Gamma, T_{\left[a, t_{0}\right]}\right)+\mathrm{V}\left(\Gamma, T_{\left[t_{0},+\infty\right)}\right) \\
& =\mathrm{V}\left(\Gamma_{1}, T_{\left[a, t_{0}\right)}\right)+d_{H}\left(\Gamma_{1}\left(s_{0}\right), \Gamma_{2}\left(t_{0}\right)\right)+\mathrm{V}\left(\Gamma_{2}, T_{\left[t_{0},+\infty\right)}\right) \\
& \leq \overrightarrow{\mathrm{V}}\left(F, T_{\left[a, t_{0}\right)}\right)+d_{H}\left(\Gamma\left(s_{0}\right), \Gamma\left(t_{0}\right)\right)+\overrightarrow{\mathrm{V}}\left(F, T_{\left[t_{0},+\infty\right)}\right) .
\end{aligned}
$$

This proves inequality (2.1) with $J_{a}\left(\Gamma, t_{0}\right)=d_{H}\left(\Gamma\left(s_{0}\right), \Gamma\left(t_{0}\right)\right)$ from (2.2).

Now, suppose $s_{0} \notin T_{\left[a, t_{0}\right)}$. Note that $s_{0}$ is a left limit point of $T_{\left[a, s_{0}\right)}$ (and so, of $T_{\left[a, t_{0}\right)}$ and $T$ as well). In fact, by the definition of $s_{0}, t<s_{0}$ for all $t \in T_{\left[a, s_{0}\right)}$, and, given $\varepsilon>0$, there is $t_{\varepsilon} \in T_{\left[a, s_{0}\right)}$ such that $s_{0}-\varepsilon<t_{\varepsilon}$. Hence $s_{0}-\varepsilon<t_{\varepsilon}<s_{0}$, i.e., $T_{\left[a, s_{0}\right)} \cap\left(s_{0}-\varepsilon, s_{0}\right) \neq \varnothing$ (and a fortiori $\left.T \cap\left(s_{0}-\varepsilon, s_{0}\right) \neq \varnothing\right)$.

Let us show that the limit in the right-hand side of (2.2) exists in $[0,+\infty)$. Since $\Gamma=\Gamma_{1}$ on $T_{\left[a, t_{0}\right)}$ and $\mathrm{V}\left(\Gamma_{1}, T_{\left[a, t_{0}\right)}\right) \leq \overrightarrow{\mathrm{V}}\left(F, T_{\left[a, t_{0}\right)}\right)$, given $t, t^{\prime} \in T_{\left[a, s_{0}\right)}$ with $t \leq t^{\prime}$, the additivity of $\mathrm{V}$ implies (note again that $a=\min T$ )

$$
\begin{aligned}
0 & \leq \mathrm{V}\left(\Gamma, T_{\left[t, t_{0}\right]}\right)-\mathrm{V}\left(\Gamma, T_{\left[t^{\prime}, t_{0}\right]}\right)=\mathrm{V}\left(\Gamma, T_{\left[t, t^{\prime}\right]}\right) \\
& =\mathrm{V}\left(\Gamma_{1}, T_{\left(-\infty, t^{\prime}\right]}\right)-\mathrm{V}\left(\Gamma_{1}, T_{(-\infty, t]}\right) .
\end{aligned}
$$


By (3.6) $)$, the right-hand side here tends to $\mathrm{V}\left(\Gamma_{1}, T_{\left[a, s_{0}\right)}\right)-\mathrm{V}\left(\Gamma_{1}, T_{\left[a, s_{0}\right)}\right)=0$ as $T \ni t, t^{\prime} \rightarrow s_{0}-0$, and so, Cauchy's criterion yields the existence of the limit.

Applying the additivity of $\mathrm{V}$ once again, we get

$$
\mathrm{V}\left(\Gamma, T_{\left[a, t_{0}\right]}\right)=\mathrm{V}\left(\Gamma, T_{[a, t]}\right)+\mathrm{V}\left(\Gamma, T_{\left[t, t_{0}\right]}\right) \quad \text { for all } t \in T_{\left[a, s_{0}\right)} .
$$

Noting that $s_{0} \notin T_{\left[a, t_{0}\right)}$ implies $T_{\left[a, s_{0}\right)}=T_{\left[a, t_{0}\right)}$, by virtue of (3.6), the limit of the first term in (4.17) as $T \ni t \rightarrow s_{0}-0$ is equal to

$$
\begin{aligned}
\lim _{T \ni t \rightarrow s_{0}-0} \mathrm{~V}\left(\Gamma, T_{[a, t]}\right) & =\lim _{T \ni t \rightarrow s_{0}-0} \mathrm{~V}\left(\Gamma_{1}, T_{(-\infty, t]}\right)=\mathrm{V}\left(\Gamma_{1}, T_{\left[a, s_{0}\right)}\right) \\
& =\mathrm{V}\left(\Gamma_{1}, T_{\left[a, t_{0}\right)}\right) \leq \overrightarrow{\mathrm{V}}\left(F, T_{\left[a, t_{0}\right)}\right) .
\end{aligned}
$$

Taking into account (2.2), it follows from (4.17) that

$$
\begin{aligned}
\mathrm{V}(\Gamma, T) & =\mathrm{V}\left(\Gamma, T_{\left[a, t_{0}\right]}\right)+\mathrm{V}\left(\Gamma, T_{\left[t_{0},+\infty\right)}\right) \\
& =\lim _{T \ni t \rightarrow s_{0}-0} \mathrm{~V}\left(\Gamma, T_{[a, t]}\right)+\lim _{T \ni t \rightarrow s_{0}-0} \mathrm{~V}\left(\Gamma, T_{\left[t, t_{0}\right]}\right)+\mathrm{V}\left(\Gamma_{2}, T_{\left[t_{0},+\infty\right)}\right) \\
& =\mathrm{V}\left(\Gamma_{1}, T_{\left[a, t_{0}\right)}\right)+J_{a}\left(\Gamma, t_{0}\right)+\mathrm{V}\left(\Gamma_{2}, T_{\left[t_{0},+\infty\right)}\right) \\
& \leq \overrightarrow{\mathrm{V}}\left(F, T_{\left[a, t_{0}\right)}\right)+J_{a}\left(\Gamma, t_{0}\right)+\overrightarrow{\mathrm{V}}\left(F, T_{\left[t_{0},+\infty\right)}\right)
\end{aligned}
$$

This proves the first inequality in (2.1).

It remains to show that if $s_{0}=t_{0}$, then $J_{a}\left(\Gamma, t_{0}\right)$ is the left jump of $\Gamma$ at $t_{0}$. Noting that, by the additivity of $\mathrm{V}$,

$$
\mathrm{V}\left(\Gamma, T_{\left[t, t_{0}\right]}\right)=\vec{v}\left(t_{0}\right)-\vec{v}(t) \quad \text { for all } t \in T_{\left[a, t_{0}\right)},
$$

and, by (3.8),

$$
\vec{v}\left(t_{0}\right)=\vec{v}\left(t_{0}-0\right)+\lim _{T \ni t \rightarrow t_{0}-0} d_{H}\left(\Gamma(t), \Gamma\left(t_{0}\right)\right),
$$

and passing to the limit as $T \ni t \rightarrow t_{0}-0$, we get

$$
\begin{aligned}
J_{a}\left(\Gamma, t_{0}\right) & =\lim _{T \ni t \rightarrow t_{0}-0} \mathrm{~V}\left(\Gamma, T_{\left[t, t_{0}\right]}\right)=\vec{v}\left(t_{0}\right)-\lim _{T \ni t \rightarrow t_{0}-0} \vec{v}(t) \\
& =\vec{v}\left(t_{0}-0\right)+\lim _{T \ni t \rightarrow t_{0}-0} d_{H}\left(\Gamma(t), \Gamma\left(t_{0}\right)\right)-\vec{v}\left(t_{0}-0\right) \\
& =\lim _{T \ni t \rightarrow t_{0}-0} d_{H}\left(\Gamma(t), \Gamma\left(t_{0}\right)\right) .
\end{aligned}
$$

This completes the proof of Theorem 1. 
Now, we are in a position to show that Theorem 1 implies Theorem A. Proof (of Theorem $\mathbf{A}$ ). Setting $T_{+}=T_{\left[t_{0},+\infty\right)}$ and $T_{-}=T_{\left(-\infty, t_{0}\right]}$, we have

$$
\overrightarrow{\mathrm{V}}\left(F, T_{+}\right) \leq \mathrm{V}\left(F, T_{+}\right) \leq \mathrm{V}(F, T) \text { and } \overleftarrow{\mathrm{V}}\left(F, T_{-}\right) \leq \mathrm{V}\left(F, T_{-}\right) \leq \mathrm{V}(F, T)
$$

Noting that $t_{0} \in T_{+} \cap T_{-}$and applying Theorem 1(a) to $F$ on $T_{+}$and Theorem 1(b) to $F$ on $T_{-}$, we obtain a set-valued selector $\Gamma_{+}: T_{+} \rightarrow \mathrm{c}(M)$ of $F$ on $T_{+}$and a set-valued selector $\Gamma_{-}: T_{-} \rightarrow \mathrm{c}(M)$ of $F$ on $T_{-}$such that

$$
\begin{gathered}
d_{H}\left(X_{0}, \Gamma_{+}\left(t_{0}\right)\right) \leq e\left(X_{0}, F\left(t_{0}\right)\right) \quad \text { and } \quad \mathrm{V}\left(\Gamma_{+}, T_{+}\right) \leq \overrightarrow{\mathrm{V}}\left(F, T_{+}\right), \\
d_{H}\left(\Gamma_{+}\left(t_{0}\right), \Gamma_{-}\left(t_{0}\right)\right) \leq e\left(\Gamma_{+}\left(t_{0}\right), F\left(t_{0}\right)\right)=0 \quad \text { and } \quad \mathrm{V}\left(\Gamma_{-}, T_{-}\right) \leq \overleftarrow{\mathrm{V}}\left(F, T_{-}\right)
\end{gathered}
$$

Noting that $\Gamma_{-}\left(t_{0}\right)=\Gamma_{+}\left(t_{0}\right)$, we set $\Gamma(t):=\Gamma_{+}(t)$ if $t \in T_{+}$, and $\Gamma(t):=\Gamma_{-}(t)$ if $t \in T_{-} \backslash\left\{t_{0}\right\}$. Clearly, $\Gamma: T \rightarrow \mathrm{c}(M)$ is a set-valued selector of $F$ on $T$, $d_{H}\left(X_{0}, \Gamma\left(t_{0}\right)\right) \leq e\left(X_{0}, F\left(t_{0}\right)\right)$ and, by the additivity of $\mathrm{V}$,

$$
\begin{aligned}
\mathrm{V}(\Gamma, T) & =\mathrm{V}\left(\Gamma, T_{+}\right)+\mathrm{V}\left(\Gamma, T_{-}\right)=\mathrm{V}\left(\Gamma_{+}, T_{+}\right)+\mathrm{V}\left(\Gamma_{-}, T_{-}\right) \\
& \leq \overrightarrow{\mathrm{V}}\left(F, T_{+}\right)+\overleftarrow{\mathrm{V}}\left(F, T_{-}\right) \\
& \leq \mathrm{V}\left(F, T_{+}\right)+\mathrm{V}\left(F, T_{-}\right)=\mathrm{V}(F, T) .
\end{aligned}
$$

This finishes the proof of Theorem $\mathrm{A}$. Note that we have shown a little bit more: inequality (4.18) holds provided $\overrightarrow{\mathrm{V}}\left(F, T_{+}\right)$and $\overleftarrow{\mathrm{V}}\left(F, T_{-}\right)$are finite.

Remark 2. If $X_{0}=\left\{x_{0}\right\} \subset M$, a (single-valued) selector of bounded variation $\Gamma: T \rightarrow M$ of $F$ on $T$ may be chosen such that $d\left(x_{0}, \Gamma\left(t_{0}\right)\right) \leq d\left(x_{0}, F\left(t_{0}\right)\right)$ and satisfying the rest of assertions in (a) and (b) of Theorem 1 (if we replace $d_{H}$ by $d$ everywhere). In order to see this, it suffices to pick only one element in the corresponding metric projection. So (cf. Step 2 in the proof of Theorem 11), choose $y_{0} \in F\left(t_{0}\right)$ such that $d\left(x_{0}, y_{0}\right)=d\left(x_{0}, F\left(t_{0}\right)\right)$, set $y_{0}^{n}:=y_{0}$, and if $i \in\{1, \ldots, n\}$ and elements $y_{i-1}^{n} \in F\left(t_{i-1}^{n}\right)$ are already chosen, pick $y_{i}^{n} \in F\left(t_{i}^{n}\right)$ such that $d\left(y_{i-1}^{n}, y_{i}^{n}\right)=d\left(y_{i-1}^{n}, F\left(t_{i}^{n}\right)\right)$. Define $\Gamma_{n}: T \rightarrow M$ (as in (4.7) and (4.8) ) by $\Gamma_{n}\left(t_{i}^{n}\right):=y_{i}^{n}$ for $i=0,1, \ldots, n$, and $\Gamma_{n}(t):=y_{i-1}^{n}$ if $t \in T \cap\left(t_{i-1}^{n}, t_{i}^{n}\right)$ and $i=1, \ldots, n$. It remains to note (for $T \subset\left[t_{0}, b\right]$ ) that

$$
\begin{aligned}
\mathrm{V}\left(\Gamma_{n}, T\right) & =\mathrm{V}\left(\Gamma_{n}, T \cap\left[t_{i-1}^{n}, t_{i}^{n}\right]\right)=\sum_{i=1}^{n} d\left(y_{i-1}^{n}, y_{i}^{n}\right) \\
& =\sum_{i=1}^{n} d\left(y_{i-1}^{n}, F\left(t_{i}^{n}\right)\right) \leq \sum_{i=1}^{n} e\left(F\left(t_{i-1}^{n}\right), F\left(t_{i}^{n}\right)\right) \leq \overrightarrow{\mathrm{V}}(F, T) .
\end{aligned}
$$

In this way, Theorem 1 above is a generalization of [17, Theorem 1], treating the existence of single-valued selectors on (connected) intervals $T \subset \mathbb{R}$. 


\section{Examples}

In examples below, we show that all assumptions in Theorem 1 are essential.

Let $(\mathbb{B},|\cdot|)$ be a Banach space with norm $|\cdot|($ e.g., $\mathbb{B}=\mathbb{R}), \mathbb{B}^{\mathbb{N}}$ be the set of all sequences $x: \mathbb{N} \rightarrow \mathbb{B}$, and $M=\ell_{1}(\mathbb{N} ; \mathbb{B})$ be the (infinite-dimensional) Banach space of all summable sequences $x \in \mathbb{B}^{\mathbb{N}}$ equipped with the norm $\|x\|:=\sum_{i=1}^{\infty}|x(i)|<+\infty$ and, hence, metric $d(x, y):=\|x-y\|$ for $x, y \in M$. Fix $u \in \mathbb{B}$ with $|u|=1$ (e.g., $u=1$ in $\mathbb{R}$ ) and, for every $n \in \mathbb{N}$, denote by $u_{n}$ the unit vector in $M$ defined as usual by $u_{n}(i)=0$ if $i \neq n$, and $u_{n}(n)=u$.

Example 1. A multifunction $F$ on $T \subset \mathbb{R}$ with (only) bounded closed values in $M$ and $\vec{V}(F, T)<+\infty$ may have no set-valued selectors $\Gamma$ satisfying

$$
\mathrm{V}\left(\Gamma, T_{\left[t_{0},+\infty\right)}\right) \leq \overrightarrow{\mathrm{V}}\left(F, T_{\left[t_{0},+\infty\right)}\right) \quad \text { or } \quad d_{H}\left(X_{0}, \Gamma\left(t_{0}\right)\right) \leq e\left(X_{0}, F\left(t_{0}\right)\right)
$$

with $t_{0} \in T$ and $X_{0} \in \mathrm{c}(M)$. In order to see this, we set $T:=[0,1]$ and $X:=$ $X_{0} \cup Y$, where $X_{0}:=\left\{u_{1}\right\}$ and $Y:=\left\{\alpha_{n} u_{n}: n \geq 2\right\}$ with $\alpha_{n}:=1+(1 / n)$, and note that $X$ and $Y$ are bounded and closed (but not compact) subsets of $M$, whereas $X_{0} \in \mathrm{c}(M)$ and $X_{0} \subset X$. Define $F: T \rightarrow\{X, Y\} \subset \mathcal{P}(M) \backslash\{\varnothing\}$ by $F(0):=X$ and $F(t):=Y$ if $0<t \leq 1$. (A similar example was given

in [3, Example 2] for $F$ with $\mathrm{V}(F, T)<+\infty$.) We have $\overrightarrow{\mathrm{V}}(F, T)=e(X, Y)$, where

$$
\begin{aligned}
e(X, Y) & =\sup _{x \in X} \inf _{y \in Y} d(x, y)=e(X \backslash Y, Y)=e\left(X_{0}, Y\right) \\
& =\inf _{n \geq 2}\left(\left|u_{1}\right|+\alpha_{n}\left|u_{n}\right|\right)=1+\inf _{n \geq 2}\left(1+\frac{1}{n}\right)=2 .
\end{aligned}
$$

Since $Y \subset X, F$ is nonincreasing on $T$ (Section 3.1), and so, $\overleftarrow{V}(F, T)=0$.

(a) Suppose $t_{0}=0$, so that $T_{\left[t_{0},+\infty\right)}=T$. We have $X_{0} \subset X=F(0)$, which implies $e\left(X_{0}, F(0)\right)=0$. Let $\Gamma: T \rightarrow \mathcal{P}(M) \backslash\{\varnothing\}$ be any set-valued selector of $F$ on $T$ such that $\Gamma(0)=X_{0}$. Since $\varnothing \neq \Gamma(1) \subset F(1)=Y, \alpha_{n} u_{n} \in \Gamma(1)$ for some $n \geq 2$. It follows that

$$
\begin{aligned}
\mathrm{V}(\Gamma, T) & \geq d_{H}(\Gamma(0), \Gamma(1)) \geq e(\Gamma(1), \Gamma(0))=e\left(\Gamma(1), X_{0}\right) \\
& \geq d\left(\alpha_{n} u_{n}, u_{1}\right)=\alpha_{n}+1>2=\overrightarrow{\mathrm{V}}(F, T) .
\end{aligned}
$$

(b) Now, suppose $0<t_{0} \leq 1$, and $\Gamma: T \rightarrow \mathcal{P}(M) \backslash\{\varnothing\}$ is a set-valued selector of $F$ on $T$. Since $\Gamma\left(t_{0}\right) \subset F\left(t_{0}\right)=Y$, we have $\alpha_{n} u_{n} \in \Gamma\left(t_{0}\right)$ for some 
$n \geq 2$, and so,

$$
\begin{aligned}
d_{H}\left(X_{0}, \Gamma\left(t_{0}\right)\right) & \geq e\left(\Gamma\left(t_{0}\right), X_{0}\right) \geq d\left(\alpha_{n} u_{n}, u_{1}\right)=\alpha_{n}+1 \\
& >2=e\left(X_{0}, Y\right)=e\left(X_{0}, F\left(t_{0}\right)\right) .
\end{aligned}
$$

(c) The effect of nonexistence of set-valued selectors in (a) and (b) above is due to the fact that $\operatorname{Pr}_{Y} X_{0}=\varnothing$ (cf. (4.5)): indeed, if $y \in Y$, then $y=\alpha_{n} u_{n}$ for some $n \geq 2$, and so, for $x \in X_{0}=\left\{u_{1}\right\}$, we have

$$
d(x, y)=d\left(u_{1}, \alpha_{n} u_{n}\right)=1+\alpha_{n}>2=e\left(X_{0}, Y\right)=d\left(u_{1}, Y\right)=d(x, Y) .
$$

Example 2. This example is more subtle than Example 1: even if $F(t)$ is bounded and closed (but not compact) at a single point $t \in T$, inequalities (5.1) may not hold in Theorem 10 (this is inspired by [13, Example 5.2]).

Let $N \in \mathbb{N}, N \geq 2$, be fixed and $\left\{\alpha_{n}\right\}_{n=1}^{\infty} \subset \mathbb{R}$ be a sequence such that

$$
\left\{\left|\alpha_{n}\right|\right\}_{n=1}^{\infty} \text { is strictly decreasing and } \inf _{n \geq N+1}\left|\alpha_{n}\right|>0
$$

(e.g., $\alpha_{n}=\alpha(n+1) / n$ with $\left.\alpha \neq 0, n \in \mathbb{N}\right)$. We set $X:=\left\{\alpha_{n} u_{n}: 1 \leq n \leq N\right\}$ and $Y:=\left\{\alpha_{n} u_{n}: n \geq N+1\right\}$. Clearly, $X \in \mathrm{c}(M)$, while $Y \notin \mathrm{c}(M)$ is bounded (by the first condition in (5.2)) and closed (by the second condition in (5.2) ) in $M$. Define $F$ on $T:=[0,1]$ by $F(t):=X$ if $0 \leq t<1$, and $F(1):=Y$. We have

$$
\overrightarrow{\mathrm{V}}(F, T)=e(X, Y)=\left|\alpha_{1}\right|+\inf _{n \geq N+1}\left|\alpha_{n}\right|
$$

and

$$
\overleftarrow{\mathrm{V}}(F, T)=e(Y, X)=\left|\alpha_{N+1}\right|+\left|\alpha_{N}\right|
$$

(a) Suppose $0 \leq t_{0}<1$ and $X_{0}:=\left\{\alpha_{1} u_{1}\right\}$, so that $T_{\left[t_{0},+\infty\right)}=\left[t_{0}, 1\right]$ and $X_{0} \subset X=F\left(t_{0}\right)$, which implies $e\left(X_{0}, F\left(t_{0}\right)\right)=0$. Let $\Gamma: T \rightarrow \mathcal{P}(M) \backslash\{\varnothing\}$ be a set-valued selector of $F$ on $T$ such that $\Gamma\left(t_{0}\right)=X_{0}$. Since $\Gamma(1) \subset F(1)=$ $Y$, we find $\alpha_{n} u_{n} \in \Gamma(1)$ for some $n \geq N+1$, and so,

$$
\begin{aligned}
\mathrm{V}\left(\Gamma,\left[t_{0}, 1\right]\right) & \geq d_{H}\left(\Gamma\left(t_{0}\right), \Gamma(1)\right) \geq e\left(\Gamma(1), X_{0}\right) \geq d\left(\alpha_{n} u_{n}, \alpha_{1} u_{1}\right)=\left|\alpha_{n}\right|+\left|\alpha_{1}\right| \\
& >\left|\alpha_{1}\right|+\inf _{i \geq N+1}\left|\alpha_{i}\right|=\overrightarrow{\mathrm{V}}(F, T)=\overrightarrow{\mathrm{V}}\left(F,\left[t_{0}, 1\right]\right) .
\end{aligned}
$$


(b) Suppose $t_{0}=1$ and $X_{0}:=\left\{\alpha_{1} u_{1}\right\}$. If $\Gamma: T \rightarrow \mathcal{P}(M) \backslash\{\varnothing\}$ is a set-valued selector of $F$ on $T$, then $\Gamma(1) \subset F(1)=Y$ implies $\alpha_{n} u_{n} \in \Gamma(1)$ for some $n \geq N+1$, and so,

$$
\begin{aligned}
d_{H}\left(X_{0}, \Gamma(1)\right) & \geq e\left(\Gamma(1), X_{0}\right) \geq d\left(\alpha_{n} u_{n}, \alpha_{1} u_{1}\right)=\left|\alpha_{n}\right|+\left|\alpha_{1}\right| \\
& >\left|\alpha_{1}\right|+\inf _{i \geq N+1}\left|\alpha_{i}\right|=e(X, Y) \geq e\left(X_{0}, Y\right)=e\left(X_{0}, F\left(t_{0}\right)\right) .
\end{aligned}
$$

(c) Clearly, $\operatorname{Pr}_{X} X_{0}=X_{0}$. The non-existence of set-valued selectors in (a) and (b) is again due to the fact that $\operatorname{Pr}_{Y} X_{0}=\varnothing$ : in fact, if $y \in Y$, then $y=\alpha_{n} u_{n}$ for some $n \geq N+1$, and so, we have, for $x \in X_{0}=\left\{\alpha_{1} u_{1}\right\}$,

$d(x, y)=d\left(\alpha_{1} u_{1}, \alpha_{n} u_{n}\right)=\left|\alpha_{1}\right|+\left|\alpha_{n}\right|>\inf _{i \geq N+1}\left(\left|\alpha_{1}\right|+\left|\alpha_{i}\right|\right)=\inf _{y \in Y} d(x, y)=d(x, Y)$.

(d) We claim that Theorem 1 (b) holds with $t_{0}=1$ and $\Gamma(1)=X_{0}$ (except that $\Gamma(1) \in \mathrm{c}(M))$ for every nonempty $X_{0} \subset Y=F(1)$.

First, observe that $\operatorname{Pr}_{X} Y_{0}=\left\{\alpha_{N} u_{N}\right\}$ for every $\varnothing \neq Y_{0} \subset Y$ (recall that $X \in \mathrm{c}(M)$, and $\operatorname{Pr}_{X} Y_{0}$ is the set of those $x \in X$, for which $d\left(y_{0}, x\right)=d\left(y_{0}, X\right)$ for some $\left.y_{0} \in Y_{0}\right)$. To see this, we set $n_{0}:=\min \left\{n \geq N+1: \alpha_{n} u_{n} \in Y_{0}\right\}$ for $Y_{0} \subset Y$. If $y_{0} \in Y_{0}$, we have $y_{0}=\alpha_{n} u_{n}$ for some $n \geq n_{0}$,

$$
d\left(y_{0}, X\right)=\inf _{x \in X} d\left(y_{0}, x\right)=\min _{1 \leq i \leq N}\left(\left|\alpha_{n}\right|+\left|\alpha_{i}\right|\right)=\left|\alpha_{n}\right|+\left|\alpha_{N}\right|,
$$

and

$$
d\left(y_{0}, x\right)=\left|\alpha_{n}\right|+\left|\alpha_{i}\right| \text { if } x=\alpha_{i} u_{i} \in X \text { for some } 1 \leq i \leq N .
$$

If $n=n_{0}$ and $i=N$, we find $y_{0}=\alpha_{n_{0}} u_{n_{0}} \in Y_{0}, x=\alpha_{N} u_{N} \in X$, and

$$
d\left(y_{0}, x\right)=d\left(\alpha_{n_{0}} u_{n_{0}}, \alpha_{N} u_{N}\right)=\left|\alpha_{n_{0}}\right|+\left|\alpha_{N}\right|=d\left(y_{0}, X\right),
$$

which implies $\alpha_{N} u_{N} \in \operatorname{Pr}_{X} Y_{0}$. Now, if $i<N$, then $\left|\alpha_{i}\right|>\left|\alpha_{N}\right|$, and so, for every $y_{0} \in Y_{0}$, we get

$$
d\left(y_{0}, x\right)=\left|\alpha_{n}\right|+\left|\alpha_{i}\right|>\left|\alpha_{n}\right|+\left|\alpha_{N}\right|=d\left(y_{0}, X\right) .
$$

Thus, $\alpha_{i} u_{i} \notin \operatorname{Pr}_{X} Y_{0}$ for all $i=1, \ldots, N-1$, and we are through.

Taking the above into account, define a set-valued selector $\Gamma$ of $F$ on $T$ by $\Gamma(t):=\left\{\alpha_{N} u_{N}\right\}$ if $0 \leq t<1$, and $\Gamma(1):=X_{0}$ with $X_{0}=Y_{0} \subset Y$. It remains to note that $n_{0} \geq N+1$ implies

$$
\begin{aligned}
\overleftarrow{\mathrm{V}}(\Gamma, T) & =e\left(Y_{0},\left\{\alpha_{N} u_{N}\right\}\right)=\sup _{y_{0} \in Y_{0}} d\left(y_{0}, \alpha_{N} u_{N}\right)=\left|\alpha_{n_{0}}\right|+\left|\alpha_{N}\right| \\
& \leq\left|\alpha_{N+1}\right|+\left|\alpha_{N}\right|=\overleftarrow{\mathrm{V}}(F, T) .
\end{aligned}
$$


Example 3. Making use of an idea from [17, Example 3.1], here we present an example of a multifunction $F: T \rightarrow \mathrm{c}(M)$ with $T:=[0,1]$ such that $\overrightarrow{\mathrm{V}}(F, T)<+\infty$ and $\overleftarrow{\mathrm{V}}(F, T)=+\infty$ (thus, Theorem 1(a) is applicable to $F$, whereas Theorem $\mathrm{A}$ is not).

Let $N \in \mathbb{N}$ and $\left\{\alpha_{n}\right\}_{n=1}^{\infty} \subset(0,+\infty)$ be a decreasing sequence such that

$$
\lim _{n \rightarrow \infty} \alpha_{n}=0 \quad \text { and } \quad \sum_{n=1}^{\infty} \alpha_{n N}=+\infty
$$

(e.g., $\left.\alpha_{n}=1 / n\right)$. Given $n \in \mathbb{N}$, we set

$$
X_{n}:=\{0\} \cup\left\{\alpha_{i} u_{i}: 1 \leq i \leq n N\right\}, \quad \text { and } \quad X_{\infty}:=\{0\} \cup\left\{\alpha_{i} u_{i}: i \in \mathbb{N}\right\} .
$$

Clearly, $X_{n} \in \mathrm{c}(M)$ for all $n \in \mathbb{N}$ and, by the first assumption in (5.3), the set $X_{\infty}$ is compact as well. Let $\left\{\tau_{n}\right\}_{n=0}^{\infty} \subset[0,1)$ be a strictly increasing sequence such that $\tau_{0}=0$ and $\lim _{n \rightarrow \infty} \tau_{n}=1$. Define $F: T \rightarrow \mathrm{c}(M)$ by the rule:

$$
F(t):=X_{n} \text { if } \tau_{n-1} \leq t<\tau_{n} \text { for all } n \in \mathbb{N} \text {, and } F(1):=X_{\infty} .
$$

Since $X_{n} \subset X_{n+1} \subset X_{\infty}$ for all $n \in \mathbb{N}, F$ is nondecreasing on $T$ (Section 3.1), and so, $\vec{V}(F, T)=0$. In order to see that $\overleftarrow{V}(F, T)=+\infty$, given $m \in \mathbb{N}$ $m \geq 2$, and partition $\pi_{m}=\left\{\tau_{n}\right\}_{n=0}^{m-1} \cup\{1\}$ of $T=[0,1]$, we find

$$
\begin{aligned}
\overleftarrow{\mathrm{V}}(F, T) & \geq \sum_{n=1}^{m-1} e\left(F\left(\tau_{n}\right), F\left(\tau_{n-1}\right)\right)+e\left(F(1), F\left(\tau_{m-1}\right)\right) \\
& =\sum_{n=1}^{m-1} e\left(X_{n+1}, X_{n}\right)+e\left(X_{\infty}, X_{m}\right),
\end{aligned}
$$

where

$$
e\left(X_{n+1}, X_{n}\right)=\sup _{n N+1 \leq k \leq(n+1) N}\left(\left|\alpha_{k}\right|+\inf _{1 \leq i \leq n N}\left|\alpha_{i}\right|\right)=\alpha_{n N+1}+\alpha_{n N}
$$

and

$$
e\left(X_{\infty}, X_{m}\right)=\sup _{k \geq m N+1}\left(\left|\alpha_{k}\right|+\inf _{1 \leq i \leq m N}\left|\alpha_{i}\right|\right)=\alpha_{m N+1}+\alpha_{m N} .
$$

It follows that the quantity (5.4) is equal to

$$
\sum_{n=1}^{m} \alpha_{n N+1}+\sum_{n=1}^{m} \alpha_{n N} \rightarrow+\infty \quad \text { as } \quad m \rightarrow \infty .
$$


Example 4. (a) By Theorem 1(a), given $X_{0} \in \mathrm{c}(M)$ such that $X_{0} \subset X_{1}=$ $F(0)$, multifunction $F$ from Example 3 has a constant set-valued selector $\Gamma: T \rightarrow \mathrm{c}(M)$ satisfying $\Gamma(0)=X_{0}$ and $\mathrm{V}(\Gamma, T) \leq \overrightarrow{\mathrm{V}}(F, T)=0$. However, if $0<t_{0} \leq 1$ and $X_{0} \subset F\left(t_{0}\right)$, there may be no set-valued selector $\Gamma$ of $F$ on $T$ such that $\Gamma\left(t_{0}\right)=X_{0}$ and $\mathrm{V}(\Gamma, T) \leq \overrightarrow{\mathrm{V}}(F, T)$. In fact, let $t_{0}=\tau_{n}$ for some $n \in \mathbb{N}$ (cf. Example 3), so that $F\left(t_{0}\right)=F\left(\tau_{n}\right)=X_{n+1}$. Suppose now that $X_{0} \subset X_{n+1} \backslash X_{1} \subset F\left(t_{0}\right), \Gamma(t) \subset F(t)$ for all $t \in T$, and $\Gamma\left(t_{0}\right)=X_{0}$. Since $\Gamma(0) \subset F(0)=X_{1}$, we have $\alpha_{i} u_{i} \in \Gamma(0)$ for some $1 \leq i \leq N$, or $0 \in \Gamma(0)$ (i.e., possibly, $\alpha_{i}=0$ ), and so,

$$
\begin{aligned}
\mathrm{V}(\Gamma, T) & \geq d_{H}\left(\Gamma\left(t_{0}\right), \Gamma(0)\right)=d_{H}\left(X_{0}, \Gamma(0)\right) \geq e\left(\Gamma(0), X_{0}\right) \\
& \geq d\left(\alpha_{i} u_{i}, X_{0}\right) \geq d\left(\alpha_{i} u_{i}, X_{n+1} \backslash X_{1}\right) \\
& =\min _{N+1 \leq k \leq(n+1) N}\left(\alpha_{i}+\alpha_{k}\right) \geq \alpha_{(n+1) N}>0=\overrightarrow{\mathrm{V}}(F, T) .
\end{aligned}
$$

(b) If $t_{0}>a=\inf T$ and $\Gamma\left(t_{0}\right)=X_{0}$, inequality $\mathrm{V}\left(\Gamma, T_{\left[a, t_{0}\right)}\right) \leq \overrightarrow{\mathrm{V}}\left(F, T_{\left[a, t_{0}\right)}\right)$ in Theorem 1(a) cannot in general be replaced by $\mathrm{V}\left(\Gamma, T_{\left[a, t_{0}\right]}\right) \leq \overrightarrow{\mathrm{V}}\left(F, T_{\left[a, t_{0}\right]}\right)$. This can be seen from Example 4 and (5.5):

$$
\mathrm{V}\left(\Gamma,\left[0, t_{0}\right]\right) \geq d_{H}\left(\Gamma\left(t_{0}\right), \Gamma(0)\right) \geq \cdots \geq \alpha_{(n+1) N}>0=\overrightarrow{\mathrm{V}}\left(F,\left[0, t_{0}\right]\right) .
$$

This observation also makes it explicit that the "jump" $J_{a}\left(\Gamma, t_{0}\right)$ is essential in the left-hand side of (2.1).

Example 5. This example is designed for Remark 1. Let $T:=[1,+\infty)$ and $F: T \rightarrow \mathrm{c}(M)$ be given by $F(t):=X_{n}$ if $n \in \mathbb{N}$ and $n \leq t<n+1$, where $X_{n}:=\left\{u_{i}: 1 \leq i \leq n\right\}$. We have $X_{n} \in \mathrm{c}(M)$, and $F(s) \subset F(t)$ for all $1 \leq s \leq$ $t<+\infty$, and so, $\overrightarrow{\mathrm{V}}(F, T)=0$. The image $F(T)=\bigcup_{n=1}^{\infty} X_{n}=\left\{u_{i}: i \in \mathbb{N}\right\}$ is bounded in $M$, but not totally bounded (i.e., cannot be covered by a finite number of balls of arbitrarily small radius). Note that $\overleftarrow{\mathrm{V}}(F, T)=+\infty$ (consider a partition $1<2<\cdots<m-1<m$ of $T$ with arbitrary $m \in \mathbb{N}$ and observe that $e\left(X_{n+1}, X_{n}\right)=2$ for all $\left.n \in \mathbb{N}\right)$. This example is easily adapted to the case when $F$ maps $[a, b)$ or $[a, b]$ into c $(M)$.

\section{Functional Inclusion $X(t) \subset F(t, X(t))$}

Assuming some interplay of the (uniform) boundedness of directional variations and (uniform) contractions, we have the following parametrized version of Banach's Contraction Theorem, extending Theorem 11.4 from [13]. 
Theorem 2. Suppose a multifunction $F: T \times \mathrm{c}(M) \rightarrow \mathrm{c}(M)$ is such that:

(a) there is a nondecreasing bounded function $\varphi: T \rightarrow \mathbb{R}$ such that $e(F(s, X), F(t, X)) \leq \varphi(t)-\varphi(s)$ for all $s, t \in T, s \leq T$, and $X \in \mathrm{c}(M) ;$

(b) there is a number $0 \leq \mu<1$ such that

$$
e(F(t, X), F(t, Y)) \leq \mu d_{H}(X, Y) \text { for all } t \in T \text { and } X, Y \in \mathrm{c}(M) ;
$$

(c) there is a multifunction $K: T \rightarrow \mathrm{c}(M)$ such that

$$
F(t, X) \subset K(t) \text { for all } t \in T \text { and } X \in \mathrm{c}(M) \text {. }
$$

If $t_{0}:=\inf T \in T$ and $X_{0} \in \mathrm{c}(M)$, then there is $X: T \rightarrow \mathrm{c}(M)$ such that (i) $\mathrm{V}(X, T) \leq \mathrm{V}(\varphi, T) /(1-\mu)<+\infty$; (ii) $X(t) \subset F(t, X(t))$ for all $t \in T$;

(iii) $d_{H}\left(X_{0}, X\left(t_{0}\right)\right) \leq e\left(X_{0}, F\left(t_{0}, X\left(t_{0}\right)\right)\right)$.

In addition, if $X_{0} \subset F\left(t_{0}, X_{0}\right)$, then (iii) can be replaced by $X\left(t_{0}\right)=X_{0}$.

Proof. First, observe that assumptions (a) and (b) and the triangle inequality for $e$ imply, for all $s, t \in T, s \leq t$, and $X, Y \in \mathrm{c}(M)$,

$$
e(F(s, X), F(t, Y)) \leq \varphi(t)-\varphi(s)+\mu d_{H}(X, Y) .
$$

We set $X_{0}(t):=X_{0}$ and $F_{0}(t):=F\left(t, X_{0}\right)$ for $t \in T$. We have $F_{0}: T \rightarrow \mathrm{c}(M)$, and assumption (a) and Lemma 1 imply $\overrightarrow{\mathrm{V}}\left(F_{0}, T\right) \leq \mathrm{V}(\varphi, T)<+\infty$. By Theorem 1(a), there is $X_{1} \equiv \Gamma: T=T_{\left[t_{0},+\infty\right)} \rightarrow \mathrm{c}(M)$ such that $X_{1}(t) \subset F_{0}(t)$ for all $t \in T, d_{H}\left(X_{0}, X_{1}\left(t_{0}\right)\right) \leq e\left(X_{0}, F_{0}\left(t_{0}\right)\right)$, and $\mathrm{V}\left(X_{1}, T\right) \leq \overrightarrow{\mathrm{V}}\left(F_{0}, T\right) \leq$ $\mathrm{V}(\varphi, T)$. In what follows we apply the standard iteration procedure. Setting $F_{1}(t):=F\left(t, X_{1}(t)\right)$ for $t \in T$, we find $F_{1}: T \rightarrow \mathrm{c}(M)$ and, by (6.1),

$$
e\left(F_{1}(s), F_{1}(t)\right) \leq \varphi(t)-\varphi(s)+\mu d_{H}\left(X_{1}(s), X_{1}(t)\right) \quad \forall s, t \in T, s \leq t .
$$

Arguing with partitions of $T$, Lemma 1 implies

$$
\overrightarrow{\mathrm{V}}\left(F_{1}, T\right) \leq \mathrm{V}(\varphi, T)+\mu \mathrm{V}\left(X_{1}, T\right) \leq(1+\mu) \mathrm{V}(\varphi, T) .
$$

Applying Theorem 11 again, we obtain $X_{2}: T \rightarrow \mathrm{c}(M)$ such that $X_{2}(t) \subset$ $F_{1}(t)$ for all $t \in T, d_{H}\left(X_{0}, X_{2}\left(t_{0}\right)\right) \leq e\left(X_{0}, F_{1}\left(t_{0}\right)\right)$, and

$$
\mathrm{V}\left(X_{2}, T\right) \leq \overrightarrow{\mathrm{V}}\left(F_{1}, T\right) \leq(1+\mu) \mathrm{V}(\varphi, T) .
$$


If $F_{2}(t):=F\left(t, X_{2}(t)\right), t \in T$, then $F_{2}: T \rightarrow \mathrm{c}(M)$ and, by (6.1),

$$
e\left(F_{2}(s), F_{2}(t)\right) \leq \varphi(t)-\varphi(s)+\mu d_{H}\left(X_{2}(s), X_{2}(t)\right) \quad \forall s, t \in T, s \leq t,
$$

and so,

$$
\overrightarrow{\mathrm{V}}\left(F_{2}, T\right) \leq \mathrm{V}(\varphi, T)+\mu \mathrm{V}\left(X_{2}, T\right) \leq\left(1+\mu+\mu^{2}\right) \mathrm{V}(\varphi, T) .
$$

Arguing by induction, we obtain the sequence $\left\{X_{n}\right\}_{n=1}^{\infty}$ of multifunctions $X_{n}: T \rightarrow \mathrm{c}(M)$ such that, given $n \in \mathbb{N}$,

$$
\begin{gathered}
X_{n}(t) \subset F_{n-1}(t):=F\left(t, X_{n-1}(t)\right) \subset K(t) \quad \text { for all } t \in T, \\
d_{H}\left(X_{0}, X_{n}\left(t_{0}\right)\right) \leq e\left(X_{0}, F_{n-1}\left(t_{0}\right)\right)=e\left(X_{0}, F\left(t_{0}, X_{n-1}\left(t_{0}\right)\right)\right), \text { and } \\
\mathrm{V}\left(X_{n}, T\right) \leq\left(\sum_{i=0}^{n-1} \mu^{i}\right) \mathrm{V}(\varphi, T) \leq \frac{1}{1-\mu} \mathrm{V}(\varphi, T) .
\end{gathered}
$$

By (6.4), the sequence $\left\{X_{n}\right\}_{n=1}^{\infty}$ is of uniformly bounded Jordan variation with respect to $d_{H}$, and so, condition (a) in Theorem $\mathrm{B}$ is satisfied, and by (6.2), the closure $\overline{\left\{X_{n}(t): n \in \mathbb{N}\right\}}$ in $\mathrm{c}(M)$ is compact for every $t \in T$, and so, condition (b) in Theorem $\mathrm{B}$ is fulfiled. By Theorem $\mathrm{B}$, a subsequence of $\left\{X_{n}\right\}_{n=1}^{\infty}$, again denoted by $\left\{X_{n}\right\}_{n=1}^{\infty}$, converges in $\mathrm{c}(M)$ pointwise on $T$ to a multifunction $X: T \rightarrow \mathrm{c}(M)$, i.e., $d_{H}\left(X_{n}(t), X(t)\right) \rightarrow 0$ as $n \rightarrow \infty$ for all $t \in T$.

We are going to verify that $X$ satisfies assertions (i), (ii), and (iii). Assertion (i) is a consequence of (6.4) and the lower semicontinuity (3.5) of V. In order to see that (ii) holds, we make use of the following inequality (cf. [13, inequality (11.7)]), which is valid for all $X, X^{\prime}, Y, Y^{\prime} \in \mathrm{c}(M)$ :

$$
\left|e(X, Y)-e\left(X^{\prime}, Y^{\prime}\right)\right| \leq d_{H}\left(X, X^{\prime}\right)+d_{H}\left(Y, Y^{\prime}\right) .
$$

In fact, given $t \in T$, (6.2) implies $e\left(X_{n}(t), F\left(t, X_{n-1}(t)\right)\right)=0$, and so, taking into account (6.5) and (6.1), we get

$$
\begin{aligned}
e(X(t), F(t, X(t))) & =\left|e(X(t), F(t, X(t)))-e\left(X_{n}(t), F\left(t, X_{n-1}(t)\right)\right)\right| \\
& \leq d_{H}\left(X(t), X_{n}(t)\right)+d_{H}\left(F(t, X(t)), F\left(t, X_{n-1}(t)\right)\right) \\
& \leq d_{H}\left(X(t), X_{n}(t)\right)+\mu d_{H}\left(X(t), X_{n-1}(t)\right) \rightarrow 0 \text { as } n \rightarrow \infty .
\end{aligned}
$$

Thus, $e(X(t), F(t, X(t)))=0$, which implies (by properties of $e$ ) assertion (ii). 
To establish (iii), we note that (cf. (6.3) and (iii))

$$
\left|d_{H}\left(X_{0}, X_{n}\left(t_{0}\right)\right)-d_{H}\left(X_{0}, X\left(t_{0}\right)\right)\right| \leq d_{H}\left(X_{n}\left(t_{0}\right), X\left(t_{0}\right)\right) \rightarrow 0 \text { as } n \rightarrow \infty,
$$

and, by virtue of (6.5) and assumption (b),

$$
\begin{aligned}
& \quad\left|e\left(X_{0}, F\left(t_{0}, X_{n-1}\left(t_{0}\right)\right)\right)-e\left(X_{0}, F\left(t_{0}, X\left(t_{0}\right)\right)\right)\right| \\
& \leq d_{H}\left(F\left(t_{0}, X_{n-1}\left(t_{0}\right)\right), F\left(t_{0}, X\left(t_{0}\right)\right)\right) \leq \mu d_{H}\left(X_{n-1}\left(t_{0}\right), X\left(t_{0}\right)\right) \rightarrow 0, n \rightarrow \infty .
\end{aligned}
$$

Passing to the limit as $n \rightarrow \infty$ in (6.3), we arrive at (iii).

Finally, suppose $X_{0} \subset F\left(t_{0}, X_{0}\right)$. Hence $X_{0} \subset F_{0}\left(t_{0}\right)$ and $e\left(X_{0}, F_{0}\left(t_{0}\right)\right)=0$. From the above, $d_{H}\left(X_{0}, X_{1}\left(t_{0}\right)\right) \leq e\left(X_{0}, F_{0}\left(t_{0}\right)\right)=0$, and so, $X_{1}\left(t_{0}\right)=X_{0}$. Since

$$
X_{0} \subset F_{0}\left(t_{0}\right)=F\left(t_{0}, X_{0}\right)=F\left(t_{0}, X_{1}\left(t_{0}\right)\right)=F_{1}\left(t_{0}\right),
$$

we find from $d_{H}\left(X_{0}, X_{2}\left(t_{0}\right)\right) \leq e\left(X_{0}, F_{1}\left(t_{0}\right)\right)=0$ that $X_{2}\left(t_{0}\right)=X_{0}$. By induction, we deduce from (6.3) that $X_{n}\left(t_{0}\right)=X_{0}$ for all $n \in \mathbb{N}$. Passing to the lmit as $n \rightarrow \infty$ in (6.3) , we get $d_{H}\left(X_{0}, X\left(t_{0}\right)\right) \leq e\left(X_{0}, F\left(t_{0}, X_{0}\right)\right)=0$, which yields $X\left(t_{0}\right)=X_{0}$.

Remark 3. If $F(t, X)=F(t)$ is independent of $X \in \mathrm{c}(M)$ (or $\mu=0)$, Theorem 2 gives back Theorem 1(a): we may set $\varphi=\vec{v}_{F}$ and $K=F$. On the other hand, if $F(t, X)=F(X)$ is independent of $t \in T$ (or $\varphi \equiv 0$ ), Theorem 2 is a consequence of Banach's Contraction Theorem (in fact, $F: \mathrm{c}(K) \rightarrow \mathrm{c}(K)$ is a contraction on compact, hence complete, metric space $\left(\mathrm{c}(K), d_{H}\right)$ with $\left.K=K\left(t_{0}\right)\right)$.

Example 6. The purpose of this example is to show that assumptions of Theorem 2 can be fulfiled. Let $M=\mathbb{B}$ be a Banach space with norm $|\cdot|$ and metric $d(x, y)=|x-y|, x, y \in M$, and $K \in \mathrm{c}(M)$. Suppose $\varphi_{0}: T \rightarrow[0,+\infty)$ is nondecreasing and $\mu:=\sup _{t \in T} \varphi_{0}(t)<1$. Define $F: T \times \mathrm{c}(M) \rightarrow \mathrm{c}(M)$ by $F(t, X):=\varphi_{0}(t) X$ for $t \in T$ and $X \in \mathrm{c}(M)$. We have

$$
e(F(s, X), F(t, X)) \leq\left(\varphi_{0}(t)-\varphi_{0}(s)\right) \max _{x \in X}|x| \quad \text { for all } s, t \in T, s \leq t,
$$

and so, condition (a) in Theorem 2 is satisfied with $\varphi(t):=\varphi_{0}(t) \max _{x \in K}|x|$, $t \in T$, for all $X \in \mathrm{c}(K)$. Furthermore, given $t \in T$ and $X, Y \in \mathrm{c}(K) \subset \mathrm{c}(M)$,

$$
e(F(t, X), F(t, Y))=\varphi_{0}(t) e(X, Y) \leq \mu d_{H}(X, Y),
$$

and so, condition (b) in Theorem 2 is satisfied. Finally, setting $K(t):=$ $\varphi_{0}(t) K$, we find $F(t, X) \subset K(t)$ for all $t \in T$ and $X \in \mathrm{c}(K)$. 
Example 7. In Example 6 , we set $\mathbb{B}:=\mathbb{R}, K:=[0,1]$, and $\varphi_{0}(t):=t$ for $t \in T:=[0,1 / 2]$ (hence $\mu=1 / 2, \varphi=\varphi_{0}$, and $\mathrm{V}(\varphi, T)=1 / 2$ ). Define $F$ by

$$
F(t, X):=(t X) \cup(1-t+t X), \quad t \in T, X \in \mathrm{c}(K) .
$$

For instance, if $X=[0,1]$, we have $F(0, X)=\{0,1\}, F(1 / 2, X)=[0,1]$, and if $0<t<1 / 2$, then $F(t, X)=[0, t] \cup[1-t, 1], F(t,[0, t])=\left[0, t^{2}\right] \cup[1-$ $\left.t, 1-t+t^{2}\right], F(t,[1-t, 1])=\left[t-t^{2}, t\right] \cup\left[1-t^{2}, 1\right]$, and so on. The iterative construction of the classical Cantor (ternary) set corresponds to $t=1 / 3$ (e.g., [20, p. 20]).

By Theorem 2, there is $X:[0,1 / 2] \rightarrow \mathrm{c}([0,1])$ such that $\mathrm{V}(X,[0,1 / 2]) \leq 1$, $X(t) \subset(t X(t)) \cup(1-t+t X(t))$ for all $t \in[0,1 / 2]$, and $X(0)=\{0,1\}$. For every $t \in(0,1 / 2)$, the compact set $X(t) \subset[0,1]$ is a Cantor-type perfect set.

Acknowledgments. The article was prepared within the framework of the Academic Fund Program at the National Research University Higher School of Economics (HSE) in 2017-2018 (grant no. 17-01-0050) and by the Russian Academic Excellence Project "5-100".

\section{References}

[1] J.-P. Aubin and A. Cellina, "Differential Inclusions: Set-Valued Maps and Viability Theory", Springer-Verlag, Berlin, 1984.

[2] J.-P. Aubin and H. Frankowska, "Set-Valued Analysis", Birkhäuser, Boston, 1990.

[3] S. A. Belov and V. V. Chistyakov, A selection principle for mappings of bounded variation, J. Math. Anal. Appl. 249 (2) (2000), 351-366.

[4] V. Berinde, M. Păcurar, The role of the Pompeiu-Hausdorff metric in fixed point theory, Creat. Math. Inform. 22 (2) (2013), 143-150.

[5] Ch. Castaing and V. Valadier, "Convex Analysis and Measurable Multifunctions", Springer, Berlin, 1977.

[6] Z. A. Chanturiya, The modulus of variation of a function and its application in the theory of Fourier series, Soviet Math. Dokl. 15 (1) (1974), 67-71.

[7] V. V. Chistyakov, "The Variation (Lecture Notes)", University of Nizhny Novgorod, Nizhny Novgorod, 1992 (in Russian).

[8] V. V. Chistyakov, On mappings of bounded variation, J. Dynam. Control Systems 3 (2) (1997), 261-289. 
[9] V. V. Chistyakov, On the theory of multivalued mappings of bounded variation of one real variable, Sbornik Math. 189 (5/6) (1998), 797-819.

[10] V. V. Chistyakov, Mappings of bounded variation with values in a metric space: generalizations, J. Math. Sci. (NY) 100 (6) (2000), 2700-2715.

[11] V. V. Chistyakov, Generalized variation of mappings with applications to composition operators and multifunctions, Positivity 5 (4) (2001), 323-358.

[12] V. V. Chistyakov, On multi-valued mappings of finite generalized variation, Math. Notes 71 (3/4) (2002), 556-575.

[13] V. V. Chistyakov, Selections of bounded variation, J. Appl. Anal. 10 (1) (2004), 1-82.

[14] V. V. Chistyakov, The optimal form of selection principles for functions of a real variable, J. Math. Anal. Appl. 310 (2) (2005), 609-625.

[15] V. V. Chistyakov and O. E. Galkin, On maps of bounded p-variation with $p>1$, Positivity 2 (1) (1998), 19-45.

[16] V. V. Chistyakov and A. Nowak, Regular Carathéodory-type selectors under no convexity assumptions, J. Funct. Anal. 225 (2) (2005), 247-262.

[17] V. V. Chistyakov and D. Repovš, Selections of bounded variation under the excess restrictions, J. Math. Anal. Appl. 331 (2) (2007), 873-885.

[18] V. V. Chistyakov and A. Rychlewicz, On the extension and generation of set-valued mappings of bounded variation, Studia Math. 153 (3) (2002), 235-247.

[19] Ş. Cobzaş, "Functional Analysis in Asymmetric Normed Spaces", Springer, Basel, 2013.

[20] K. Goebel and W. A. Kirk, "Topics in Metric Fixed Point Theory", Cambridge Univ. Press, Cambridge, 1990.

[21] C. Goffman, N. Nishiura and D. Waterman, "Homeomorphisms in Analysis", Amer. Math. Soc., Providence, RI, 1997.

[22] J. Guričan and P. Kostyrko, On Lipschitz selections of Lipschitz multifunctions, Acta Math. Univ. Comenian. 46/47 (1985), 83-85.

[23] H. Hermes, Existence and properties of solutions of $\dot{x} \in R(t, x)$, In: "Advances in Differential and Integral Equations", J. A. Nohel (ed.), (Univ. Wisconsin, 1968), Studies in Appl. Math. SIAM 5 (1969), 188-193.

[24] H. Hermes, On continuous and measurable selections and the existence of solutions of generalized differential equations, Proc. Amer. Math. Soc. 29 (3) (1971), 535-542. 
[25] N. Kikuchi and Y. Tomita, On the absolute continuity of multi-functions and orientor fields, Funkcial. Ekvac. 14 (3) (1971), 161-170.

[26] I. Kupka, Continuous multifunction from $[-1,0]$ to $\mathbb{R}$ having no continuous selection, Publ. Math. Debrecen 48 (3/4) (1996), 367-370.

[27] I. Kupka, Continuous selections for Lipschitz multifunctions, Acta Math. Univ. Comenian. 74 (1) (2005), 133-141.

[28] E. Michael, Continuous selections, I, II, III, Ann. Math. 63 (2) (1956), 361-382; 64 (3) (1956), 562-580; 65 (2) (1957), 375-390.

[29] B. S. Mordukhovich, "Approximation Methods in Problems of Optimization and Control", Nauka, Moscow, 1988 (in Russian).

[30] D. Pompeiu, Sur la continuité des fonctions de variables complexes, Ann. Fac. Sci. de Toulouse $2^{e}$ sér. 7 (3) (1905), 265-315.

[31] D. Repovš and P. V. Semenov, "Continuous Selections of Multivalued Mappings", Kluwer Acad. Publ., Dordrecht, 1998.

[32] B. Satco, Continuous dependence results for set-valued measure differential problems, Electron. J. Qualitat. Theory Diff. Equat. 79 (2015), 1-15.

[33] W.A. Ślȩzak, Concerning continuous selectors for multifunctions with nonconvex values, Probl. Mat. 9 (1987), 85-104.

[34] Yu. V. Tretyachenko, "Pointwise selection principles for functions of one and several real variables", Candidate Dissertation, Nizhny Novgorod (2010) (in Russian).

[35] Q.J. Zhu, Single-valued representation of absolutely continuous setvalued mappings, Kexue Tongbao 31 (7) (1986), 443-446 (in Chinese). 